\title{
SINGULAR INVARIANT HYPERFUNCTIONS ON THE SQUARE MATRIX SPACE AND THE ALTERNATING MATRIX SPACE
}

\author{
MASAKAZU MURO*
}

\begin{abstract}
Fundamental calculations on singular invariant hyperfunctions on the $n \times n$ square matrix space and on the $2 n \times 2 n$ alternating matrix space are considered in this paper. By expanding the complex powers of the determinant function or the Pfaffian function into the Laurent series with respect to the complex parameter, we can construct singular invariant hyperfunctions as their Laurent expansion coefficients. The author presents here the exact orders of the poles of the complex powers and determines the exact supports of the Laurent expansion coefficients. By applying these results, we prove that every quasirelatively invariant hyperfunction can be expressed as a linear combination of the Laurent expansion coefficients of the complex powers and that every singular quasi-relatively invariant hyperfunction is in fact relatively invariant on the generic points of its support. In the last section, we give the formula of the Fourier transforms of singular invariant tempered distributions.
\end{abstract}

\section{$\S 1$. Introduction}

Let $\boldsymbol{V}:=\operatorname{Mat}_{n}(\mathbb{R})$ be the real vector space of $n \times n$ real matrices. The real connected algebraic group $G:=\mathbf{G L}_{n}(\mathbb{R})^{+} \times \mathbf{S L}_{n}(\mathbb{R})$ acts on $\operatorname{Mat}_{n}(\mathbb{R})$ algebraically by

$$
(g, x) \longmapsto g \cdot x:=g_{1} x^{t} g_{2}
$$

with $g:=\left(g_{1}, g_{2}\right) \in \boldsymbol{G}$ and $x \in \boldsymbol{V}$. Here, $\mathbf{G L}_{n}(\mathbb{R})^{+}:=\left\{g \in \operatorname{Mat}_{n}(\mathbb{R}) \mid\right.$ $\operatorname{det}(g)>0\}$ and $\mathbf{S L}_{n}(\mathbb{R}):=\left\{g \in \operatorname{Mat}_{n}(\mathbb{R}) \mid \operatorname{det}(g)=1\right\}$. Then the pair $(\boldsymbol{G}, \boldsymbol{V})$ is a regular prehomogeneous vector space with an irreducible relative invariant $P(x):=\operatorname{det}(x)$ and $\boldsymbol{V}$ decomposes into a finite number of $\boldsymbol{G}$-orbits. This is the first object of this paper. Next, let $\boldsymbol{V}:=\operatorname{Alt}_{2 n}(\mathbb{R})$

Received December 4, 2001.

2000 Mathematics Subject Classification: Primary 22E45, 20G20; Secondary 11E39.

* Supported in part by The Mitsubishi Foundation and by Grant-in-Aid for Scientific Research, (C)(2)13640163 Ministry of Education, Culture, Sports, Science and Technology, Japan 
be the real vector space of $2 n \times 2 n$ real alternating matrices. The real connected algebraic group $G:=\mathbf{G L}_{2 n}(\mathbb{R})^{+}$acts on $\operatorname{Alt}_{2 n}(\mathbb{R})$ algebraically by

$$
(g, x) \longmapsto g \cdot x:=g x^{t} g
$$

with $g \in \boldsymbol{G}$ and $x \in \boldsymbol{V}$. Then the pair $(\boldsymbol{G}, \boldsymbol{V})$ is a regular prehomogeneous vector space with an irreducible relative invariant $P(x):=\operatorname{Pf}(x)$ and $\boldsymbol{V}$ decomposes into a finite number of $\boldsymbol{G}$-orbits. Here $\operatorname{Pf}(x)$ stands for the Pfaffian of the alternating matrix $x$ which is a polynomial on $\operatorname{Alt}_{2 n}(\mathbb{R})$ given as a square root of $\operatorname{det}(x)$ taking the value $\operatorname{Pf}\left(J_{n}\right)=(-1)^{n(n-1) / 2}$ for $J_{n}:=\left(\begin{array}{cc}0_{n} & I_{n} \\ -I_{n} & 0_{n}\end{array}\right)$. This is the second object in this paper.

These two prehomogeneous vector spaces have some common properties. Both of two have two open $\boldsymbol{G}$-orbits, one is $\boldsymbol{V}_{+}:=\{x \in \boldsymbol{V} \mid P(x)>0\}$ and the other is $\boldsymbol{V}_{-}:=\{x \in \boldsymbol{V} \mid P(x)<0\}$. We define the complex power of the relative invariant $P(x)$ by

$$
|P(x)|_{ \pm}^{s}:= \begin{cases}|P(x)|^{s} & \text { if } x \in \boldsymbol{V}_{ \pm} \\ 0 & \text { if } x \notin \boldsymbol{V}_{ \pm}\end{cases}
$$

for $s \in \mathbb{C}$. Then it is well known that the integral $\int|P(x)|_{ \pm}^{s} f(x) d x$ is absolutely convergent for all the rapidly decreasing function $f(x)$ provided that the real part $\Re(s)$ of $s \in \mathbb{C}$ is non-negative. In addition, $|P(x)|_{ \pm}^{s}$ can be regarded as a tempered distribution — and hence a hyperfunction on $\boldsymbol{V}$ with a holomorphic parameter $s \in \mathbb{C}$ and it is extended to the whole complex plane as a meromorphic function in $s \in \mathbb{C}$. The poles of $P^{[\vec{a}, s]}(x)$ belong to the set $\mathbb{Z}_{<0}:=\{-1,-2, \ldots\}$. We define the linear combination of $|P(x)|_{ \pm}^{s}$ by

$$
P^{[\vec{a}, s]}(x):=a_{+}|P(x)|_{+}^{s}+a_{-}|P(x)|_{-}^{s}
$$

for $\vec{a}:=\left(a_{+}, a_{-}\right) \in \mathbb{C}^{2}$ and $s \in \mathbb{C}$, which is a relatively invariant hyperfunction under the action of $\boldsymbol{G}$. Namely we have

$$
P^{[\vec{a}, s]}(g \cdot x)=\chi(g)^{s} P^{[\vec{a}, s]}(x)
$$

for all $g \in \boldsymbol{G}$ with $\chi(g)=\operatorname{det}\left(g_{1}\right)$ when $\boldsymbol{V}=\operatorname{Mat}_{n}(\mathbb{R})$ or with $\chi(g)=$ $\operatorname{det}(g)$ when $\boldsymbol{V}=\operatorname{Alt}_{2 n}(\mathbb{R})$. For a fixed complex number $\lambda \in \mathbb{C}$, we call a hyperfunction $f(x) \chi^{\lambda}$-invariant if it satisfies

$$
f(g \cdot x)=\chi(g)^{\lambda} f(x)
$$


for all $g \in \boldsymbol{G}$. If $\lambda \notin \mathbb{Z}_{<0}$, then $P^{[\vec{a}, \lambda]}(x)$ is well-defined and it is a $\chi^{\lambda}$ invariant hyperfunction.

Let $\boldsymbol{G}^{1}:=\{g \in \boldsymbol{G} \mid P(g \cdot x)=P(x)\}$. Then $P^{[\vec{a}, s]}(x)$ is a $\boldsymbol{G}^{1}$-invariant hyperfunction. Namely $\boldsymbol{G}^{1}=\mathbf{S L}_{n}(\mathbb{R}) \times \mathbf{S L}_{n}(\mathbb{R})$ for $\boldsymbol{V}=\operatorname{Mat}_{n}(\mathbb{R})$ and $\boldsymbol{G}^{1}=\mathbf{S L}_{2 n}(\mathbb{R})$ for $\boldsymbol{V}=\operatorname{Alt}_{2 n}(\mathbb{R})$. If $P^{[\vec{a}, s]}(x)$ has a pole at $s=s_{0}$, then hyperfunctions appearing as Laurent expansion coefficients in the principal part of the Laurent expansion of $P^{[\vec{a}, s]}(x)$ at $s=s_{0}$ are $G^{1}$-invariant and their supports are contained in the singular set $\boldsymbol{S}:=\{x \in \boldsymbol{V} \mid P(x)=0\}$. A hyperfunction is called singular if its support is contained in $\boldsymbol{S}$. We can prove that any singular $\boldsymbol{G}^{1}$-invariant tempered distribution is written as a finite linear combination of the Laurent expansion coefficients of negative degree of $P^{[\vec{a}, s]}(x)$ (see Propositions 4.1 and 4.2 ) by using the results in Muro [9] and Muro [10].

The purpose of this paper is to investigate some properties of hyperfunctions - especially singular ones - on $\boldsymbol{V}$ which are invariant under the action of $\boldsymbol{G}^{1}$ via the hyperfunctions appearing as Laurent expansion coefficients of $P^{[\vec{a}, s]}(x)$ - especially of negative degree - and give the formula of Fourier transforms. More concretely we shall give answers to the following problems in this paper.

Problem 1.1. (Fundamental problems) We call the following problems fundamental problems for a complex power function $P^{[\vec{a}, s]}(x)$.

1. Give the exact orders of poles of $P^{[\vec{a}, s]}(x)$ (Theorems 6.3 and 6.4 in Section 6).

2. Give the exact supports of the hyperfunctions appearing in the Laurent expansion coefficients of $P^{[\vec{a}, s]}(x)$ especially of negative degree (Theorems 7.1 and 7.2 in Section 7).

3. Give a basis of singular invariant hyperfunctions by using the Laurent expansion coefficients appearing in the principal parts of the Laurent expansion coefficients at poles of $P^{[\vec{a}, s]}(x)$.

4. Give explicit formulas of the Fourier transforms of singular $\mathbf{G}^{1}$ invariant tempered distributions (Theorems 9.3, 9.4, 9.7 and 9.8 in Section 9).

Through the precise investigation of the properties of Laurent expansion coefficients of $P^{[\vec{a}, s]}(x)$, we have discovered some interesting facts about singular invariant hyperfunctions as by-products. Namely every hyperfunction appearing as a Laurent expansion coefficient of $P^{[\vec{a}, s]}(x)$ at $s=\lambda$ is 
a quasi- $\chi^{\lambda}$-invariant hyperfunction (Proposition 3.3). For the definition of quasi- $\chi^{\lambda}$-invariance, see Definition 2.1. Conversely, we see that any quasi$\chi^{\lambda}$-invariant hyperfunction is given as a linear combination of Laurent expansion coefficients at $s=\lambda$, and in particular, any singular quasi- $\chi^{\lambda}$ invariant hyperfunction is a linear combination of Laurent expansion coefficients at $s=\lambda$ of negative degree (Theorem 8.1). In addition, we prove that a singular quasi- $\chi^{\lambda}$-invariant hyperfunction is in fact $\chi^{\lambda}$-invariant at a point near which the support is a non-singular variety (Theorem 8.2).

Furthermore, we investigate bi-singular $G^{1}$-invariant tempered distributions on $\operatorname{Mat}_{n}(\mathbb{R})$. We say that a $\boldsymbol{G}^{1}$-invariant tempered distribution $f(x)$ is bi-singular if both $f(x)$ and its Fourier transform $f(x)^{\vee}:=\int f(x)$ $\exp (-\sqrt{-1}\langle x, y\rangle) d x$ are singular. It is known that a $\boldsymbol{G}^{1}$-invariant measure on a single singular orbit (i.e., a $\boldsymbol{G}$-orbit in $\boldsymbol{S}$ ) on $\operatorname{Mat}_{n}(\mathbb{R})$ can be viewed as a tempered distribution and its Fourier transform is also a $\boldsymbol{G}^{1}$-invariant measure on a singular orbit provided that it is not a delta-function supported on the origin, and hence it is a bi-singular $G^{1}$-invariant tempered distribution (see, for example, Rubenthaler [17]). As a corollary to the formulas of the Fourier transforms, we prove that there are bi-singular $G^{1}$ invariant tempered distributions on $\boldsymbol{V}=\operatorname{Mat}_{n}(\mathbb{R})$ and we can determine all of them in Corollary 9.5, which shows that there are many other bisingular $\boldsymbol{G}^{1}$-invariant tempered distributions on $\boldsymbol{V}=\operatorname{Mat}_{n}(\mathbb{R})$. In particular, we see in Corollary 9.6 that every $\chi^{\lambda}$-invariant tempered distribution on $\boldsymbol{V}=\operatorname{Mat}_{n}(\mathbb{R})$ is singular if $\lambda=-2,-3, \ldots,-n$ and hence it is bisingular if $\lambda=-2,-3, \ldots,-n+2$. On the other hand, it is seen that there are no bi-singular $\boldsymbol{G}^{1}$-invariant tempered distributions on $\boldsymbol{V}=\operatorname{Alt}_{2 n}(\mathbb{R})$ in Corollary 9.9.

Hyperfunctions given as complex powers of polynomials are often called "local zeta functions" or "local zeta distributions" (for example, see SatoShintani [20], Shintani [21], Satake [19], Igusa [5]) and, in particular number theorists have been studying these objects especially on non-archimedean fields (for example, see Igusa [5], Saito [18]). Fundamental problems for zeta functions are the computations of functional equations, the determination of the poles and the computation of the principal part of the poles. We may say that this paper gives solutions to the fundamental problems of the local zeta functions on the archimedean field $\mathbb{R}$ for two examples studied here.

The author thinks that exact analysis of invariant hyperfunctions never has been fully developed. Further examples will be given in the future pa- 
pers. Micro-local analysis seems to be inevitable to the calculus of invariant hyperfunctions and it enables us to give a definite answer to the fundamental problems.

Acknowledgement. The author expresses his sense of gratitude to Professor A. Gyoja for his kindhearted advices.

\section{§2. Quasi-relatively invariant hyperfunctions}

In this section we describe some fundamental properties of the prehomogeneous vector spaces that we will analyze in this paper. We consider the prehomogeneous vector space

$$
\begin{aligned}
(\boldsymbol{G}, \boldsymbol{V}) & :=\left(\mathbf{G L}_{n}(\mathbb{R})^{+} \times \mathbf{S L}_{n}(\mathbb{R}), \operatorname{Mat}_{n}(\mathbb{R})\right) \\
(\operatorname{resp} .(\boldsymbol{G}, \boldsymbol{V}) & \left.:=\left(\mathbf{G L}_{2 n}(\mathbb{R})^{+}, \operatorname{Alt}_{2 n}(\mathbb{R})\right)\right) .
\end{aligned}
$$

The algebraic group $\boldsymbol{G}$ acts on $\boldsymbol{V}$ by

$$
\begin{aligned}
(g, x) & \longmapsto g \cdot x:=g_{1} x^{t} g_{2} \\
\text { (resp. }(g, x) & \left.\longmapsto g \cdot x:=g x^{t} g\right)
\end{aligned}
$$

for $(g, x) \in \boldsymbol{G} \times \boldsymbol{V}$. The Lie algebra $\mathfrak{G}$ of $\boldsymbol{G}$ is $\mathfrak{g l}_{n}(\mathbb{R}) \oplus \mathfrak{s l}_{n}(\mathbb{R})\left(\right.$ resp. $\left.\mathfrak{g l}_{2 n}(\mathbb{R})\right)$ and the Lie algebra $\mathfrak{G}^{1}$ of $\boldsymbol{G}^{1}$ is $\mathfrak{s l}_{n}(\mathbb{R}) \oplus \mathfrak{s l}_{n}(\mathbb{R})\left(\right.$ resp. $\left.\mathfrak{s l}_{2 n}(\mathbb{R})\right)$, where $\mathfrak{g l}_{n}(\mathbb{R})$ and $\mathfrak{s l}_{n}(\mathbb{R})$ are Lie algebras of $\mathbf{G L}_{n}(\mathbb{R})$ and $\mathbf{S L}_{n}(\mathbb{R})$, respectively. The infinitesimal action of (7) is

$$
\begin{aligned}
(A, x) & \longmapsto A \cdot x:=A_{1} x+x^{t} A_{2} \\
(\operatorname{resp} .(A, x) & \left.\longmapsto A \cdot x:=A x+x^{t} A\right)
\end{aligned}
$$

with $A=\left(A_{1}, A_{2}\right) \in \mathfrak{G}=\mathfrak{g l}_{n}(\mathbb{R}) \oplus \mathfrak{s l}_{n}(\mathbb{R})\left(\right.$ resp. $A \in \mathfrak{G}=\mathfrak{g l}_{2 n}(\mathbb{R})$ ). Let $\boldsymbol{V}^{*}$ be the dual space of $\boldsymbol{V}$. We define the canonical bilinear form on $(x, y) \in \boldsymbol{V} \times \boldsymbol{V}^{*}$ by

$$
\langle x, y\rangle:=\operatorname{tr}\left(x^{t} y\right) \quad\left(\text { resp. }\langle x, y\rangle:=\frac{1}{2} \operatorname{tr}\left(x^{t} y\right)\right) .
$$

We can identify $\boldsymbol{V}$ and $\boldsymbol{V}^{*}$ by $\langle-,-\rangle$. The contragredient representation with respect to $\langle-,-\rangle$ is given by

$$
\begin{aligned}
(g, y) & \longmapsto g^{*} \cdot y:={ }^{t} g_{1}^{-1} y g_{2}^{-1} \\
\text { (resp. }(g, y) & \left.\longmapsto g^{*} \cdot y:={ }^{t} g^{-1} y g^{-1}\right)
\end{aligned}
$$


and its infinitesimal action is

$$
\begin{aligned}
(A, y) & \longmapsto A^{*} \cdot y:=-{ }^{t} A_{1} y-y A_{2} \\
(\operatorname{resp} .(A, y) & \left.\longmapsto A^{*} \cdot y:=-{ }^{t} A y-y A\right)
\end{aligned}
$$

The vector space $\boldsymbol{V}$ decomposes into a finite number of $\boldsymbol{G}$-orbits. Among these orbits,

$$
\boldsymbol{V}_{+}:=\{x \in \boldsymbol{V} \mid P(x)>0\}, \quad \boldsymbol{V}_{-}:=\{x \in \boldsymbol{V} \mid P(x)<0\}
$$

are open $\boldsymbol{G}$-orbits. The set $\boldsymbol{S}:=\{x \in \boldsymbol{V} \mid P(x)=0\}$ is a $\boldsymbol{G}$-invariant subset of $\boldsymbol{V}$ and it decomposes into

$$
\boldsymbol{S}:=\bigsqcup_{i=1}^{n} \boldsymbol{S}_{i}
$$

where

$$
\boldsymbol{S}_{i}:=\{x \in \boldsymbol{V} \mid \operatorname{rank}(x)=n-i\}
$$

when $\boldsymbol{V}$ is a square matrix space, and

$$
\boldsymbol{S}_{i}:=\{x \in \boldsymbol{V} \mid \operatorname{rank}(x)=2(n-i)\}
$$

when $\boldsymbol{V}$ is an alternating matrix space. It is easily seen that each $\boldsymbol{S}_{i}$ is a $\boldsymbol{G}^{1}$-orbit for $i=1,2, \ldots, n$. The open set $\boldsymbol{V}-\boldsymbol{S}$ is sometimes denoted by $\boldsymbol{S}_{0}$.

The continuous characters of $\boldsymbol{G}$ to $\mathbb{C}^{\times}$are given by

$$
\chi^{s}(g):=\operatorname{det}\left(g_{1}\right)^{s} \quad\left(\operatorname{resp} \cdot \chi^{s}(g):=\operatorname{det}(g)^{s}\right)
$$

with $s \in \mathbb{C}$. The infinitesimal character $\delta \chi(A):=d \chi(\exp (t A)) /\left.d t\right|_{t=0}$ of $\chi$ is

$$
\delta \chi(A)=\operatorname{tr}\left(A_{1}\right) \quad(\operatorname{resp} . \delta \chi(A)=\operatorname{tr}(A))
$$

DEFINITION 2.1. (relative invariance) Let $v(x)$ be a hyperfunction on $\boldsymbol{V}$ and let $\chi^{s}$ be the character defined by (14) with a complex number $s \in \mathbb{C}$.

1. We say that $v(x)$ is $\chi^{s}$-invariant if it satisfies

$$
v(g \cdot x)=\chi(g)^{s} v(x)
$$


for all $g \in \boldsymbol{G}$. A hyperfunction which is $\chi^{s}$-invariant with some complex number $s \in \mathbb{C}$ is called relatively invariant and we say that the character $\chi^{s}$ is the corresponding character. This condition is equivalent to saying that $v(x)$ satisfies the equations of infinitesimal equations:

$$
(\langle A \cdot x, \partial\rangle-s \delta \chi(A)) v(x)=0
$$

for all $A \in \mathfrak{G}$.

2. We say that $v(x)$ is quasi- $\chi^{s}$-invariant if there exists a non-negative integer $k$ and it satisfies

$$
\left(\left\langle A_{0} \cdot x, \partial\right\rangle-s \delta \chi\left(A_{0}\right)\right) \cdots\left(\left\langle A_{k} \cdot x, \partial\right\rangle-s \delta \chi\left(A_{k}\right)\right) v(x)=0
$$

for all $A_{0}, A_{1}, \ldots, A_{k} \in \mathfrak{G}$. A hyperfunction which is quasi- $\chi^{s}$-invariant with some complex number $s \in \mathbb{C}$ is called quasi-relatively invariant and $\chi^{s}$ is called the corresponding character. We call the complex number $s$ the relative-degree. If $v(x)$ satisfies (17) then we say that $v(x)$ is a quasi$\chi^{s}$-invariant hyperfunction of quasi-relative-degree $k$. In addition, if $v(x)$ satisfies

$$
\left(\left\langle A_{0} \cdot x, \partial\right\rangle-s \delta \chi\left(A_{0}\right)\right) \cdots\left(\left\langle A_{k-1} \cdot x, \partial\right\rangle-s \delta \chi\left(A_{k-1}\right)\right) v(x) \neq 0,
$$

for some $A_{0}, A_{1}, \ldots, A_{k-1} \in \mathfrak{G}$, then we say that $v(x)$ has proper quasirelative-degree $k$.

Proposition 2.1. A hyperfunction $v(x)$ on $\boldsymbol{V}$ is quasi- $\chi^{\lambda}$-invariant of quasi-relative-degree $k \in \mathbb{Z}_{\geq 0}$ if and only if $v(x)$ is $\mathfrak{G}^{1}$-invariant, i.e., $\langle A \cdot x, \partial\rangle v(x)=0$ for all $A \in \mathfrak{G}^{1}$, and

$$
\left(\left\langle A_{0} \cdot x, \partial\right\rangle-\lambda \delta \chi\left(A_{0}\right)\right)^{k+1} v(x)=0
$$

for some $A_{0} \in \mathfrak{G}$ with $\delta \chi\left(A_{0}\right) \neq 0$. In particular, $v(x)$ has proper quasirelative-degree $k$ if and only if

$$
\left(\left\langle A_{0} \cdot x, \partial\right\rangle-\lambda \delta \chi\left(A_{0}\right)\right)^{k} v(x) \neq 0,
$$

holds in addition to (18).

Proof. We denote by $\vartheta_{\lambda}(A):=(\langle A \cdot x, \partial\rangle-\lambda \delta \chi(A))$. Then it is easily checked that

$$
\left[\vartheta_{\lambda}(A), \vartheta_{\lambda}(B)\right]=\vartheta_{\lambda}([A, B])=\langle[A, B] \cdot x, \partial\rangle
$$


for all $A, B \in \mathfrak{G}$ since $\delta \chi([A, B])=0$ for $[A, B] \in \mathfrak{G}^{1}$.

First we prove that $v(x)$ is $\mathfrak{G}^{1}$-invariant and satisfies $(18)$ if $v(x)$ is quasi- $\chi^{\lambda}$-invariant of quasi-relative-degree $k$. Let $A \in \mathfrak{G}^{1}$. Since $\mathfrak{G}^{1}$ is a semi-simple Lie algebra, we have $\mathfrak{G}^{1}=\left[\mathfrak{G}^{1}, \mathfrak{G}^{1}\right]$. Then there exist a finite number of $B^{i}, C^{i} \in \mathfrak{G}^{1}$ satisfying $A=\sum_{i}\left[B^{i}, C^{i}\right]$. Then we have

$$
\vartheta_{\lambda}(A)=\vartheta_{\lambda}\left(\sum_{i}\left[B^{i}, C^{i}\right]\right)=\sum_{i} \vartheta_{\lambda}\left(\left[B^{i}, C^{i}\right]\right)=\sum_{i}\left[\vartheta_{\lambda}\left(B^{i}\right), \vartheta_{\lambda}\left(C^{i}\right)\right] .
$$

Therefore, we see that there exist a finite number of $A_{1}^{j}, A_{2}^{j} \in \mathfrak{G}^{1}$ satisfying

$$
\vartheta_{\lambda}(A)=\sum_{j} \vartheta_{\lambda}\left(A_{1}^{j}\right) \vartheta_{\lambda}\left(A_{2}^{j}\right)
$$

Repeating this operation to $\vartheta_{\lambda}\left(A_{i}^{j}\right)$ on the right hand side $k$ times, we see that $\vartheta_{\lambda}(A)$ is written as the form

$$
\vartheta_{\lambda}(A)=\sum_{j} \vartheta_{\lambda}\left(A_{1}^{j}\right) \cdots \vartheta_{\lambda}\left(A_{k+1}^{j}\right)
$$

by using a finite number of $A_{1}^{j}, \ldots, A_{k+1}^{j} \in \mathfrak{G}^{1}$. Then, for all $A \in \mathfrak{G}^{1}$, we have

$$
\vartheta_{\lambda}(A) v(x)=\sum_{j} \vartheta_{\lambda}\left(A_{1}^{j}\right) \cdots \vartheta_{\lambda}\left(A_{k+1}^{j}\right) v(x)=0
$$

if $v(x)$ is quasi- $\chi^{\lambda}$-invariant of quasi-relative-degree $k$. This shows that $v(x)$ is $\mathfrak{G}^{1}$-invariant. The equation (18) is clear from the definition.

Next we prove the converse. We have only to show that

$$
\vartheta_{\lambda}\left(A_{1}\right) \cdots \vartheta_{\lambda}\left(A_{k+1}\right) v(x)=0
$$

for all $A_{1}, \ldots, A_{k+1} \in \mathfrak{G}$ under the assumption that $v(x)$ is $\mathfrak{G}^{1}$-invariant and (18) holds. For each $A_{i}(i=1, \ldots, k+1)$, there exist $c_{i} \in \mathbb{R}$ and $B_{i} \in \mathfrak{G}^{1}$ satisfying $A_{i}=c_{i} A_{0}+B_{i}$. Indeed, we have only to put $c_{i}:=\delta \chi\left(A_{i}\right) / \delta \chi\left(A_{0}\right)$ and $B_{i}:=A_{i}-c_{i} A_{0}$. Then we have

$$
\vartheta_{\lambda}\left(A_{1}\right) \cdots \vartheta_{\lambda}\left(A_{k+1}\right)=\prod_{i=1}^{k+1} \vartheta_{\lambda}\left(c_{i} A_{0}+B_{i}\right)=\prod_{i=1}^{k+1}\left(c_{i} \vartheta_{\lambda}\left(A_{0}\right)+\vartheta_{\lambda}\left(B_{i}\right)\right) .
$$

By expanding the right hand side and arranging it, we can write as

$$
\vartheta_{\lambda}\left(A_{1}\right) \cdots \vartheta_{\lambda}\left(A_{k+1}\right)=\left(\prod_{i=1}^{k+1} c_{i}\right) \vartheta_{\lambda}\left(A_{0}\right)^{k+1}+\sum_{j} U_{j}(x, \partial) \vartheta_{\lambda}\left(F_{j}\right)
$$


where $\left\{F_{j}\right\}$ is a basis of $\mathfrak{G}^{1}$. Since $v(x)$ is $\mathfrak{G}^{1}$-invariant and (18) holds from the assumption, we have $\vartheta_{\lambda}\left(F_{j}\right) v(x)=0$ and $\vartheta_{\lambda}\left(A_{0}\right)^{k+1} v(x)=0$, and hence we have $\vartheta_{\lambda}\left(A_{1}\right) \cdots \vartheta_{\lambda}\left(A_{k+1}\right) v(x)=0$.

(19) can be proved in the same way.

Typical relatively invariant hyperfunction is the complex power function $P^{[\vec{a}, s]}(x)$ defined by (4). If $\lambda$ is not a negative integer, then $\left.P^{[\vec{a}, s]}(x)\right|_{s=\lambda}$ $=P^{[\vec{a}, \lambda]}(x)$ is a $\chi^{\lambda}$-invariant hyperfunction. More generally, for any complex number $\lambda \in \mathbb{C}$, each Laurent expansion coefficient of $P^{[\vec{a}, s]}(x)$ at $s=\lambda$ is a quasi- $\chi^{\lambda}$-invariant hyperfunction (see Proposition 3.3).

We define odd and even invariant hyperfunctions on $\boldsymbol{V}$. We consider the case of the square matrix space $\boldsymbol{V}=\operatorname{Mat}_{n}(\mathbb{R})$ (resp. the alternating matrix space $\left.\boldsymbol{V}:=\operatorname{Alt}_{2 n}(\mathbb{R})\right)$. We set

$$
\begin{aligned}
g_{0} & :=\left(\begin{array}{cc}
I_{n-1} & 0 \\
0 & -1
\end{array}\right) \times I_{n} \in \mathbf{G L}_{n}(\mathbb{R}) \times \mathbf{G L}_{n}(\mathbb{R}) \\
\text { resp. } g_{0} & \left.:=\left(\begin{array}{ccc}
I_{2 n-2} & 0 \\
0 & 0 & 1
\end{array}\right) \in \mathbf{G L}_{2 n}(\mathbb{R})\right) .
\end{aligned}
$$

Definition 2.2. (even and odd functions) For a $\boldsymbol{G}^{1}$-invariant hyperfunction $f(x)$, we say that $f(x)$ is even if $f\left(g_{0} \cdot x\right)=f(x)$ and that $f(x)$ is odd if $f\left(g_{0} \cdot x\right)=-f(x)$.

Let $f(x)$ be a $\boldsymbol{G}^{1}$-invariant hyperfunction. Defining even and odd functions by

$$
f_{+}(x):=\frac{1}{2}\left(f(x)+f\left(g_{0} \cdot x\right)\right) \quad \text { and } \quad f_{-}(x):=\frac{1}{2}\left(f(x)-f\left(g_{0} \cdot x\right)\right),
$$

respectively, we have

$$
f(x)=f_{+}(x)+f_{-}(x)
$$

Namely we have the following proposition.

Proposition 2.2. Any $\boldsymbol{G}^{1}$-invariant (resp. quasi-relatively invariant) hyperfunction is written as a sum of an odd $\boldsymbol{G}^{1}$-invariant (resp. quasirelatively invariant) hyperfunction and an even $\boldsymbol{G}^{1}$-invariant (resp. quasirelatively invariant) hyperfunction uniquely. 


\section{$\S 3$. Some properties of complex powers of the relative invariants}

In this section we shall study the hyperfunction $P^{[\vec{a}, s]}(x)$, which is introduced by (4) and is called complex powers of the relative invariant $P(x)$.

By defining the two dimensional vectors

$$
\vec{e}_{+}:=\left(\begin{array}{l}
1 \\
0
\end{array}\right), \quad \text { and } \quad \vec{e}_{-}:=\left(\begin{array}{l}
0 \\
1
\end{array}\right)
$$

we have

$$
P^{\left[\vec{e}_{+}, s\right]}(x)=|P(x)|_{+}^{s}, \quad \text { and } \quad P^{\left[\vec{e}_{-}, s\right]}(x)=|P(x)|_{-}^{s},
$$

We define the two vectors

$$
\overrightarrow{\text { even }}:=\left(\begin{array}{l}
1 \\
1
\end{array}\right)=\vec{e}_{+}+\vec{e}_{-}, \quad \overrightarrow{\text { odd }}:=\left(\begin{array}{c}
1 \\
-1
\end{array}\right)=\vec{e}_{+}-\vec{e}_{-}
$$

and the two linear combinations of complex powers

$$
\begin{aligned}
|P(x)|_{\text {even }}^{s} & :=P^{[\overrightarrow{\mathrm{even}}, s]}(x)=|P(x)|_{+}^{s}+|P(x)|_{-}^{s}, \\
|P(x)|_{\text {odd }}^{s} & :=P^{[\overrightarrow{\mathrm{ddd}}, s]}(x)=|P(x)|_{+}^{s}-|P(x)|_{-}^{s} .
\end{aligned}
$$

Then it is easy to see that $|P(x)|_{\text {even }}^{s}$ is an even $\chi^{s}$-invariant hyperfunction and that $|P(x)|_{\text {odd }}^{s}$ is an odd $\chi^{s}$-invariant hyperfunction.

Remember the well-known formulas

$$
\operatorname{det}(\partial) \operatorname{det}(x)^{s+1}=(s+1)(s+2) \cdots(s+n) \operatorname{det}(x)^{s}
$$

on $\boldsymbol{V}=\operatorname{Mat}_{n}(\mathbb{R})$, and

$$
\operatorname{Pf}(\partial) \operatorname{Pf}(x)^{s+1}=(s+1)(s+3) \cdots(s+2 n-1) \operatorname{Pf}(x)^{s}
$$

on $\boldsymbol{V}=\operatorname{Alt}_{2 n}(\mathbb{R})$. Here $\operatorname{det}(\partial)$ is the determinant of the $n \times n$ matrix whose $(i, j)$-entry is $\partial / \partial x_{i j}$, and $\operatorname{Pf}(\partial)$ is the Pfaffian of the $2 n \times 2 n$ alternating matrix whose $(i, j)$-entry is $\partial / \partial x_{i j}$. They are proved originally as the Capelli's identity (see Weyl [22], Rais [15]). By using these formulas, we have the following proposition, which is proved in a well-known way in the theory of analytic continuation of complex powers of polynomials. See for example Bernstein [1] or Igusa [5, Theorem 5.3.1]. 
Proposition 3.1. We have:

1. $P^{[\vec{a}, s]}(x)$ is an absolutely convergent tempered distribution with a holomorphic parameter $s \in \mathbb{C}$ if the real part $\Re(s)$ is non-negative.

2. The poles of $P^{[\vec{a}, s]}(x)$ are located at $s \in \mathbb{Z}_{<0}:=\{-1,-2, \ldots\}$.

3. Let

$$
P H O(\lambda):= \begin{cases}k & \text { at } \lambda=-k(k=1,2, \ldots, n-1), \\ n & \text { at } \lambda=-k(k=n, n+1, \ldots), \\ 0 & \text { otherwise }\end{cases}
$$

if $\boldsymbol{V}=\operatorname{Mat}_{n}(\mathbb{R})$, and let

$$
P H O(\lambda):= \begin{cases}\left\lfloor\frac{k+1}{2}\right\rfloor & \text { at } \lambda=-k(k=1,2, \ldots, 2 n-2), \\ n & \text { at } \lambda=-k(k=2 n-1,2 n, \ldots), \\ 0 & \text { otherwise, }\end{cases}
$$

if $\boldsymbol{V}=\operatorname{Alt}_{n}(\mathbb{R})$. We call $\mathrm{PHO}(\lambda)$ the possible highest order of the pole of $P^{[\vec{a}, s]}(x)$ at $s=\lambda$ and the order of pole of $P^{[\vec{a}, s]}(x)$ at $s=\lambda$ is at most $\operatorname{PHO}(\lambda)$.

We define the Laurent expansion coefficients of the Laurent expansion of $P^{[\vec{a}, s]}(x)$ with respect to the complex parameter. It is a crucial object of this paper.

Definition 3.1. (Laurent expansion coefficients) Let $P^{[\vec{a}, s]}(x)$ be the hyperfunction defined by (4) with a meromorphic parameter $s \in \mathbb{C}$ and let $\lambda \in \mathbb{C}$ be a fixed point.

1. For a two dimensional non-zero complex vector $\vec{a} \in \mathbb{C}^{2}$, we define $o(\vec{a}, \lambda) \in \mathbb{Z}$ by

$$
o(\vec{a}, \lambda):=\text { the order of pole of } P^{[\vec{a}, s]}(x) \text { at } s=\lambda,
$$

and we define $o(\overrightarrow{0}, \lambda)=-\infty$.

2. For a fixed $\vec{a} \in \mathbb{C}^{2}$ and $\lambda \in \mathbb{C}$, we denote by

$$
P^{[\vec{a}, s]}(x)=\sum_{k \in \mathbb{Z}} P_{k}^{[\vec{a}, \lambda]}(x)(s-\lambda)^{k}
$$

the Laurent expansion of $P^{[\vec{a}, s]}(x)$ at $s=\lambda$ and we call $P_{k}^{[\vec{a}, \lambda]}(x)$ the Laurent expansion coefficient of degree $k$ of $P^{[\vec{a}, s]}(x)$ at $s=\lambda$. In particular, 
$P_{-o(\vec{a}, \lambda)}^{[\vec{a}, \lambda]}(x)$ is the lowest degree non-zero term among the Laurent expansion coefficients of $P^{[\vec{a}, s]}(x)$ at $s=\lambda$.

Then the following proposition is easily verified and we omit the proof.

Proposition 3.2. Let $P_{k}^{[\vec{a}, \lambda]}(x)$ be the Laurent expansion coefficient of degree $k$ of $P^{[\vec{a}, s]}(x)$ at $s=\lambda$, which is defined in (24).

1. Each $P_{k}^{[\vec{a}, \lambda]}(x)$ is a $\boldsymbol{G}^{1}$-invariant hyperfunction.

2. Each $P_{k}^{[\vec{a}, \lambda]}(x)$ is real analytic on $\boldsymbol{V}-\boldsymbol{S}$.

3. For any $\lambda \in \mathbb{C}$ and $k \in \mathbb{Z}, P_{k}^{[\vec{a}, \lambda]}(x)$ is linear with respect to $\vec{a}$.

4. If $P_{k}^{[\vec{a}, \lambda]}\left(x_{0}\right) \neq 0$ for some point $x_{0} \in \boldsymbol{V}_{+}\left(\right.$resp. $\left.\boldsymbol{V}_{-}\right)$, then it is non-zero at every point in $\boldsymbol{V}_{+}\left(\right.$resp. $\left.\boldsymbol{V}_{-}\right)$.

5. $\operatorname{Supp}\left(P_{k}^{[\vec{a}, \lambda]}(x)\right) \subset \boldsymbol{S}$ if $k<0$.

6. $P_{k}^{[\vec{a}, \lambda]}(x)=0$ if $k<-o(\vec{a}, \lambda)$.

Now we prove that the Laurent expansion coefficients $P_{k}^{[\vec{a}, \lambda]}(x)(k \in \mathbb{Z})$ are quasi-relatively invariant hyperfunctions.

Proposition 3.3. Let $\lambda$ be a complex number and let $P_{k}^{[\vec{a}, \lambda]}(x)$ be the Laurent expansion coefficient of degree $k$ of $P^{[\vec{a}, s]}(x)$ at $s=\lambda$ defined by (24). Then, for an integer $q=0,1,2, \ldots$,

$$
P_{-o(\vec{a}, \lambda)+q}^{[\vec{a}, \lambda]}(x)
$$

is a non-zero quasi- $\chi^{\lambda}$-invariant hyperfunction of proper quasi-relative-degree $q$.

Proof. Consider the Laurent expansion

$$
P^{[\vec{a}, s]}(x)=\sum_{k \in \mathbb{Z}} P_{k}^{[\vec{a}, \lambda]}(x)(s-\lambda)^{k} .
$$

Let $A_{0}$ be an element of $\mathfrak{G}$ with $\delta \chi\left(A_{0}\right)=0$. Then we have

$$
\left(\left\langle A_{0} \cdot x, \partial\right\rangle-\lambda \delta \chi\left(A_{0}\right)\right) P^{[\vec{a}, s]}(x)=\left\langle A_{0} \cdot x, \partial\right\rangle P^{[\vec{a}, s]}(x)=0,
$$

and hence we have

$$
\left(\left\langle A_{0} \cdot x, \partial\right\rangle-\lambda \delta \chi\left(A_{0}\right)\right) P_{k}^{[\vec{a}, \lambda]}(x)=0,
$$


for all $A_{0} \in \mathfrak{G}$ with $\delta \chi\left(A_{0}\right)=0$ and for all $k \in \mathbb{Z}$. Next, let $A_{0}$ be an element of $\mathfrak{G}$ satisfying $\delta \chi\left(A_{0}\right) \neq 0$ (for example, the identity matrix operator). Then we have

$$
\begin{aligned}
\delta \chi & \left(A_{0}\right)^{-1}\left(\left\langle A_{0} \cdot x, \partial\right\rangle-\lambda \delta \chi\left(A_{0}\right)\right) P^{[\vec{a}, s]}(x) \\
& =\sum_{k \in \mathbb{Z}} \delta \chi\left(A_{0}\right)^{-1}\left(\left\langle A_{0} \cdot x, \partial\right\rangle-\lambda \delta \chi\left(A_{0}\right)\right) P_{k}^{[\vec{a}, \lambda]}(x)(s-\lambda)^{k} \\
& =(s-\lambda) P^{[\vec{a}, s]}(x) \\
& =\sum_{k \in \mathbb{Z}} P_{k}^{[\vec{a}, \lambda]}(x)(s-\lambda)^{k+1}
\end{aligned}
$$

and hence we have

$$
\delta \chi\left(A_{0}\right)^{-1}\left(\left\langle A_{0} \cdot x, \partial\right\rangle-\lambda \delta \chi\left(A_{0}\right)\right) P_{k}^{[\vec{a}, \lambda]}(x)=P_{k-1}^{[\vec{a}, \lambda]}(x) .
$$

This means that $P_{k}^{[\vec{a}, \lambda]}(x) \neq 0$ if $P_{k-1}^{[\vec{a}, \lambda]}(x) \neq 0$.

On the other hand, we see from the definition that

$$
P_{-o(\vec{a}, \lambda)}^{[\vec{a}, \lambda]}(x) \neq 0 \quad \text { and } \quad P_{-o(\vec{a}, \lambda)-1}^{[\vec{a}, \lambda]}(x)=0 .
$$

Then we have

$$
\left(\delta \chi\left(A_{0}\right)^{-1}\left(\left\langle A_{0} \cdot x, \partial\right\rangle-\lambda \delta \chi\left(A_{0}\right)\right)\right)^{q} P_{-o(\vec{a}, \lambda)+q}^{[\vec{a}, \lambda]}(x)=P_{-o(\vec{a}, \lambda)}^{[\vec{a}, \lambda]}(x) \neq 0
$$

and

$$
\left(\delta \chi\left(A_{0}\right)^{-1}\left(\left\langle A_{0} \cdot x, \partial\right\rangle-\lambda \delta \chi\left(A_{0}\right)\right)\right)^{q+1} P_{-o(\vec{a}, \lambda)+q}^{[\vec{a}, \lambda]}(x)=P_{-o(\vec{a}, \lambda)-1}^{[\vec{a}, \lambda]}(x)=0 .
$$

This shows that $P_{-o(\vec{a}, \lambda)+q}^{[\vec{a}, \lambda]}(x)$ is a non-zero quasi- $\chi^{\lambda}$-invariant hyperfunction of proper quasi-relative-degree $q$.

The converse of this proposition is true and the proof will be given in Theorem 8.1.

\section{$\S 4$. Holonomic systems characterizing relatively invariance}

In this section, we introduce the holonomic system $\mathcal{M}_{\lambda}$ whose solutions are $\chi^{\lambda}$-invariant hyperfunctions on $\boldsymbol{V}$ and describe the structure of the characteristic variety $\operatorname{ch}\left(\mathcal{M}_{\lambda}\right)$. We omit the proof here since they are easily verified in the same way as the proof of the calculation of the characteristic varieties in Muro [8] and Muro [10]. 
We denote the system of linear differential equations by

$$
\mathcal{M}_{\lambda}:(\langle A \cdot x, \partial\rangle-\lambda \delta \chi(A)) f(x)=0 \quad \text { for all } A \in \mathfrak{G}
$$

Then $\mathcal{M}_{\lambda}$ is a holonomic system, for the characteristic variety of $\mathcal{M}_{\lambda}$ is given by

$$
\operatorname{ch}\left(\mathcal{M}_{\lambda}\right)=\bigcup_{i=0}^{n} \Lambda_{i}
$$

where $\Lambda_{i}:=\overline{T_{\boldsymbol{S}_{i}}^{*} \boldsymbol{V}}$. Here, $\overline{T_{\boldsymbol{S}_{i}}^{*} \boldsymbol{V}}$ means the closure of the conormal bundle of the variety $\boldsymbol{S}_{i}$. Each $\Lambda_{i}$ is an irreducible $\boldsymbol{G}$-invariant Lagrangian subvariety in $T^{*} \boldsymbol{V}=\boldsymbol{V} \times \boldsymbol{V}^{*}$, on which the group $\boldsymbol{G}$ acts in the manner given by (7) and (10). Each $\Lambda_{i}$ has the following two $G$-orbits $\Lambda_{i}^{ \pm}$. When $\boldsymbol{V}=\operatorname{Mat}_{n}(\mathbb{R})$, they are

$$
\Lambda_{i}^{ \pm}:=\boldsymbol{G} \cdot\left(\left(\begin{array}{ll}
I_{n-i} & \\
& 0_{i}
\end{array}\right),\left(\begin{array}{ll}
0_{n-i} & \\
& I_{i}^{ \pm}
\end{array}\right)\right)
$$

where $I_{i}=I_{i}^{+}$is the $i \times i$ identity matrix and $I_{i}^{-}:=\left(\begin{array}{ll}I_{i-1} & \\ & -1\end{array}\right)$. When $\boldsymbol{V}=\operatorname{Alt}_{2 n}(\mathbb{R})$, they are

$$
\Lambda_{i}^{ \pm}:=\boldsymbol{G} \cdot\left(\left(\begin{array}{ll}
J_{n-i} & \\
& 0_{2 i}
\end{array}\right),\left(\begin{array}{ll}
0_{2(n-i)} & \\
& J_{i}^{ \pm}
\end{array}\right)\right)
$$

where $J_{i}=J_{i}^{+}:=\left(\begin{array}{cc}0_{i} & I_{i} \\ -I_{i} & 0_{i}\end{array}\right)$ and $J_{i}^{-}:=\left(\begin{array}{cc}0_{i} & I_{i}^{-} \\ -I_{i}^{-} & 0_{i}\end{array}\right)$. The subset $\Lambda_{i}^{\circ}:=$ $\Lambda_{i}^{+} \sqcup \Lambda_{i}^{+}=\Lambda_{i}-\bigcup_{i \neq j} \Lambda_{j}$ is a dense subset in $\Lambda_{i}$. If $|i-j|=1$, then $\Lambda_{i}$ and $\Lambda_{j}$ has an intersection of codimension one. If $|i-j|>1$, then $\Lambda_{i}$ and $\Lambda_{j}$ has an intersection of codimension $>1$. The intersection $\Lambda_{i} \cap \Lambda_{i+1}$ has a dense $\boldsymbol{G}$-orbit in it, which is generated by

$$
\begin{gathered}
\left(\left(\begin{array}{ll}
I_{n-i-1} & \\
& 0_{i+1}
\end{array}\right),\left(\begin{array}{ll}
0_{n-i} & \\
& I_{i}
\end{array}\right)\right) \text { when } \boldsymbol{V}=\operatorname{Mat}_{n}(\mathbb{R}) \\
\left(\left(\begin{array}{ll}
J_{n-i-1} & \\
& 0_{2 i}
\end{array}\right),\left(\begin{array}{ll}
0_{2(n-i)} & \\
& J_{i}
\end{array}\right)\right) \quad \text { when } \boldsymbol{V}=\operatorname{Alt}_{2 n}(\mathbb{R}) .
\end{gathered}
$$

The following propositions were proved in Muro [10, Theorem 5.6] and Muro [10, Theorem 5.7], respectively. 
Proposition 4.1. Let $\lambda$ be an arbitrary complex number. Then any hyperfunction solution to $\mathcal{M}_{\lambda}$ belongs to the vector space generated by

$$
\left\{P_{-o(\vec{a}, \lambda)}^{[\vec{a}, \lambda]}(x) \mid \vec{a} \in \mathbb{C}^{2}\right\} .
$$

(Here, we define $P_{-o(\overrightarrow{0}, \lambda)}^{[\overrightarrow{0}, \lambda]}(x):=0$ since $P^{[\overrightarrow{0}, s]}(x) \equiv 0$.)

Proposition 4.2. Any singular $\boldsymbol{G}^{1}$-invariant tempered distribution is written as a linear combination of Laurent expansion coefficients of $P^{[\vec{a}, s]}(x)$ of negative degree at $s=-1,-2, \ldots$.

\section{§5. Principal symbols of the hyperfunction solutions to $\mathcal{M}_{\lambda}$}

In this section we review the real principal symbol $\sigma_{\Lambda_{i}^{\epsilon}}(u(s, x))$ on each Lagrangian orbit $\Lambda_{i}^{\epsilon}$ - or we simply call principal symbol - of a hyperfunction $u(s, x)$ with a meromorphic parameter $s \in \mathbb{C}$ provided that $\left.u(s, x)\right|_{s=\lambda}$ - or $\left.(s-\lambda)^{k} u(s, x)\right|_{s=\lambda}$ if $u(s, x)$ has a pole of order $k$ at $s=\lambda$ - is a solution to the holonomic system $\mathcal{M}_{\lambda}$ for each complex number $\lambda \in \mathbb{C}$. We present it by using the coefficient function $c_{i}^{\epsilon}(s)$, which is a meromorphic function in $s \in \mathbb{C}$. Then the computation of the poles and the Laurent expansion coefficients of the coefficient function vector $\left(c_{i}^{\epsilon}(s)\right)_{i=0,1, \ldots, n, \epsilon= \pm}$ is equivalent to the computation of those of $u(s, x)$. The situation is almost the same as that of the case of invariant hyperfunctions on the real symmetric matrix space, which we considered in Muro [11]. We give here some results on the relations between the hyperfunction solution $u(s, x)$ and its principal symbol $\sigma_{\Lambda_{i}^{\epsilon}}(u(s, x))$ without proof since the proof is almost the same as the one in Muro [11].

We shall give a canonical basis of the principal symbol following the theory in Kashiwara-Miwa [7] and Muro [8]. Let $\Lambda_{i}^{\circ}$ be the open subset defined above and let $\Lambda_{i}^{\epsilon}(\epsilon= \pm)$ be a connected component of $\Lambda_{i}^{\circ}$. We define a non-zero real analytic section $\Omega_{i}^{\epsilon}(s)(\epsilon= \pm)$ of the sheaf of half volume element on $\Lambda_{i}^{\epsilon}$ by

$$
\Omega_{i}^{\epsilon}(s):=\left|P_{\Lambda_{i}^{\epsilon}}(x, y)\right|^{s} \sqrt{\left|\omega_{\Lambda_{i}^{\epsilon}}(x, y)\right|} .
$$

Then the section $\Omega_{i}^{\epsilon}(s)$ depends on $s \in \mathbb{C}$ holomorphically. Here, we set

$$
\begin{gathered}
P_{\Lambda_{i}^{\epsilon}}(x, y):=P\left(\pi_{W}(x, y)\right) /\left.\left(\left.\sigma(x, y)\right|_{W}\right)^{m_{\Lambda_{i}}}\right|_{\Lambda_{i}^{\epsilon}}, \\
\omega_{\Lambda_{i}^{\epsilon}}(x, y):=\frac{\pi_{W}^{-1}(|d x|) \wedge d \sigma(x, y)}{\sigma(x, y)^{\mu_{\Lambda_{i}}}} /\left.d \sigma(x, y)\right|_{\Lambda_{i}^{\epsilon}},
\end{gathered}
$$


where $\sigma:=\sigma(x, y)$ is a function on $\boldsymbol{V} \times \boldsymbol{V}^{*}$ defined by $\sigma:=\langle x, y\rangle / n ; \pi_{W}$ is the projection map from the subvariety

$$
W:=\left\{(x, y) \in T^{*} \boldsymbol{V} \mid\langle A x, y\rangle=0 \text { for all } A \in \mathfrak{G}^{1}\right\} \subset \boldsymbol{V} \times \boldsymbol{V}^{*}
$$

to $\boldsymbol{V}$, where $\mathfrak{G}^{1}:=\{A \in \mathfrak{G} \mid \delta \chi(A)=0\} ; m_{\Lambda_{i}}$ and $\mu_{\Lambda_{i}}$ are the constants such that (33) and (34) are non-zero on $\Lambda_{i}^{\epsilon}$, respectively. We define $P(x):=$ $\operatorname{det}(x)$ (resp. $P(x):=\operatorname{Pf}(x)$ ) and $|d x|$ is a non-zero volume element on $\boldsymbol{V}$ defined by

$$
\begin{aligned}
|d x|: & =\left|\bigwedge_{1 \leq i, j \leq n} d x_{i j}\right| \\
(\text { resp. }|d x|:= & \left.\bigwedge_{1 \leq i<j \leq 2 n} d x_{i j} \mid\right)
\end{aligned}
$$

with

$$
\begin{gathered}
x=\left(\begin{array}{cccc}
x_{11} & x_{12} & \cdots & x_{1 n} \\
x_{21} & x_{22} & \cdots & x_{2 n} \\
\vdots & \vdots & \ddots & \vdots \\
x_{n 1} & x_{n 2} & \cdots & x_{n n}
\end{array}\right) \in \boldsymbol{V} \\
\text { resp. } \left.x=\left(\begin{array}{cccc}
0 & x_{1,2} & \cdots & x_{1,2 n} \\
-x_{1,2} & 0 & \cdots & x_{2,2 n} \\
\vdots & \vdots & \ddots & \vdots \\
-x_{1,2 n} & -x_{2,2 n} & \cdots & 0
\end{array}\right) \in \boldsymbol{V}\right)
\end{gathered}
$$

when $\boldsymbol{V}$ is the $n \times n$ square matrix space (resp. the $2 n \times 2 n$ alternating matrix space). We have $m_{\Lambda_{i}}=i$ and $\mu_{\Lambda_{i}}=i^{2}\left(\operatorname{resp} . m_{\Lambda_{i}}=i\right.$ and $\mu_{\Lambda_{i}}=i(2 i-1)$ ) in the case of square matrices (resp. alternating matrices). We call the homogeneous degree of (32) with respect to $y$ the order of $\mathcal{M}_{s}$ on $\Lambda_{i}$ and denote it by order $\Lambda_{i}$. In our cases, we have

$$
\operatorname{order}_{\Lambda_{i}}= \begin{cases}-i s-\left(i^{2} / 2\right) & \text { when } \boldsymbol{V}=\operatorname{Mat}_{n}(\mathbb{R}) \\ -i s-i(2 i-1) & \text { when } \boldsymbol{V}=\operatorname{Alt}_{2 n}(\mathbb{R})\end{cases}
$$

Proposition 5.1. Let $u(s, x)$ be a microfunction with a meromorphic parameter $s \in \mathbb{C}$. We suppose that $\left.u(s, x)\right|_{s=\lambda}-$ or $\left.(s-\lambda)^{k} u(s, x)\right|_{s=\lambda}$ if 
$u(s, x)$ has a pole of order $k$ at $s=\lambda$ - is a solution to the holonomic system $\mathcal{M}_{\lambda}$ for each complex number $\lambda \in \mathbb{C}$. Let $\Lambda_{i}^{\epsilon}$ be a connected component in $\Lambda_{i}^{\circ}$. Then we have:

1. The principal symbol $\sigma_{\Lambda_{i}^{\epsilon}}(u(s, x))$ is written as a constant multiple of the real analytic section of $\Omega_{i}^{\epsilon}(s) / \sqrt{|d x|}$ :

$$
\sigma_{\Lambda_{i}^{\epsilon}}(u(s, x))=c_{i}^{\epsilon}(s) \cdot \Omega_{i}^{\epsilon}(s) / \sqrt{|d x|} .
$$

Conversely, if every constant multiplication term $c_{i}^{\epsilon}(s)$ is given on each $\Lambda_{i}^{\epsilon}$, then the corresponding microfunction solution $u(s, x)$ satisfying (39) is determined uniquely provided that it exists.

2. Each $c_{i}^{\epsilon}(s)$ is a holomorphic (resp. meromorphic) function in $s \in \mathbb{C}$, if and only if $u(s, x)$ depends on $s \in \mathbb{C}$ holomorphically (resp. meromorphically) on each $\Lambda_{i}^{\epsilon}$.

Remark 5.1. The definition and some fundamental properties of hyperfunctions (resp. microfunctions) with a meromorphic parameter $s \in \mathbb{C}$ is given in Kashiwara, Kawai and Kimura [6, Chapter 3, Section 8]. In short, it is a hyperfunction (resp. microfunction) which satisfies the CauchyRiemann equation with respect to $s \in \mathbb{C}$ after regularizing the poles. For example, $P^{[\vec{a}, s]}(x)$ is a typical hyperfunction with a meromorphic parameter $s \in \mathbb{C}$.

Proof. The proof is the same as the proof of Muro [11, Proposition 3.3].

We consider hyperfunction solutions to $\mathcal{M}_{\int}$ of the form

$$
P^{[\vec{a}, s]}(x):=a_{+}|P(x)|_{+}^{s}+a_{-}|P(x)|_{-}^{s}
$$

introduced in (4). Since $P^{[\vec{a}, s]}(x)$ is a hyperfunction with a meromorphic parameter $s \in \mathbb{C}$, the microfunction image $\operatorname{sp}\left(P^{[\vec{a}, s]}(x)\right)$, where sp is the isomorphic map sp : $\mathcal{B}_{\boldsymbol{V}} \simeq \pi_{*}\left(\mathcal{C}_{\boldsymbol{V}}\right)$, and its principal symbols $\sigma_{\Lambda_{i}^{\epsilon}}\left(\mathbf{s p}\left(P^{[\vec{a}, s]}(x)\right)\right)$ depend on $s \in \mathbb{C}$ meromorphically. Here, $\mathcal{B}_{\boldsymbol{V}}$ and $\mathcal{C}_{\boldsymbol{V}}$ are the sheaves of hyperfunctions on $\boldsymbol{V}$ and microfunctions on $T^{*} \boldsymbol{V}$, respectively, and $\pi_{*}\left(\mathcal{C}_{\boldsymbol{V}}\right)$ is the direct image of the sheaf of microfunctions on $T^{*} \boldsymbol{V}$ to $\boldsymbol{V}$. We often denote the principal symbol by $\sigma_{\Lambda_{i}^{\epsilon}}\left(P^{[\vec{a}, s]}(x)\right)$ instead of $\sigma_{\Lambda_{i}^{\epsilon}}\left(\mathbf{s p}\left(P^{[\vec{a}, s]}(x)\right)\right)$ for the simplicity. 
Definition 5.1. (Coefficient functions) Let

$$
\sigma_{\Lambda_{i}^{\epsilon}}\left(P^{[\vec{a}, s]}(x)\right):=c_{i}^{\epsilon}(\vec{a}, s) \Omega_{i}^{\epsilon}(s) / \sqrt{|d x|}
$$

with $c_{i}^{\epsilon}(\vec{a}, s)$ being a meromorphic function in $s \in \mathbb{C}$. We call $c_{i}^{\epsilon}(\vec{a}, s)$ a coefficient function or simply a coefficient of $P^{[\vec{a}, s]}(x)$ on $\Lambda_{i}^{\epsilon}$ with respect to the canonical basis

$$
\Omega_{i}^{\epsilon}(s) / \sqrt{|d x|} .
$$

In particular, we see easily $c_{0}^{\epsilon}(\vec{a}, s)=a_{\epsilon}(\epsilon= \pm)$. It is easily checked that the coefficient functions $c_{i}^{\epsilon}(\vec{a}, s)$ depend on $\vec{a} \in \mathbb{C}^{n+1}$ linearly and on $s \in \mathbb{C}$ meromorphically.

We give the analytic relations (43) and (44) combining the coefficient functions of a hyperfunction solution with a meromorphic parameter $s \in \mathbb{C}$.

Proposition 5.2. The coefficient functions on $\Lambda_{i-1}^{\circ}$ and $\Lambda_{i}^{\circ}$ have the following relation for $i=1,2, \ldots, n$.

1. (the case of square matrices)

$$
\begin{aligned}
& {\left[\begin{array}{l}
c_{i}^{+}(\vec{a}, s) \\
c_{i}^{-}(\vec{a}, s)
\end{array}\right]=\frac{\Gamma(s+i)}{\sqrt{2 \pi}}} \\
& \times\left[\begin{array}{ll}
\exp \left(-\frac{\pi}{2} \sqrt{-1}(s+i)\right) & \exp \left(+\frac{\pi}{2} \sqrt{-1}(s+i)\right) \\
\exp \left(+\frac{\pi}{2} \sqrt{-1}(s+i)\right) & \exp \left(-\frac{\pi}{2} \sqrt{-1}(s+i)\right)
\end{array}\right]\left[\begin{array}{c}
c_{i-1}^{+}(\vec{a}, s) \\
c_{i-1}^{-}(\vec{a}, s)
\end{array}\right] .
\end{aligned}
$$

2. (the case of alternating matrices)

$$
\begin{aligned}
& {\left[\begin{array}{l}
c_{i}^{+}(\vec{a}, s) \\
c_{i}^{-}(\vec{a}, s)
\end{array}\right]=\frac{\Gamma(s+2 i-1)}{\sqrt{2 \pi}}} \\
& \quad \times\left[\begin{array}{ll}
\exp \left(-\frac{\pi}{2} \sqrt{-1}(s+2 i-1)\right) & \exp \left(+\frac{\pi}{2} \sqrt{-1}(s+2 i-1)\right) \\
\exp \left(+\frac{\pi}{2} \sqrt{-1}(s+2 i-1)\right) & \exp \left(-\frac{\pi}{2} \sqrt{-1}(s+2 i-1)\right)
\end{array}\right] \\
& \quad \times\left[\begin{array}{l}
c_{i-1}^{+}(\vec{a}, s) \\
c_{i-1}^{-}(\vec{a}, s)
\end{array}\right] .
\end{aligned}
$$

Proof. Consider the intersections of codimension one among the irreducible Lagrangian subvarieties in $\operatorname{ch}\left(\mathcal{M}_{s}\right)$. Then only $\Lambda_{i-1}$ and $\Lambda_{i}$ have an intersection of codimension one for $i=1,2, \ldots, n$. From Kashiwara's 
formula (Kashiwara-Miwa [7, Formula 3.6 in p. 142]), we have the relation of the coefficient functions $c_{i-1}^{\epsilon}(\vec{a}, s)$ and $c_{i}^{\epsilon}(\vec{a}, s)$, which is given by

$$
\begin{aligned}
& {\left[\begin{array}{c}
c_{i}^{+}(\vec{a}, s) \\
c_{i}^{-}(\vec{a}, s)
\end{array}\right]=\frac{\Gamma(\alpha(s))}{\sqrt{2 \pi}}\left[\begin{array}{cc}
\exp \left(-\frac{\pi}{2} \sqrt{-1}(\alpha(s))\right) & \exp \left(+\frac{\pi}{2} \sqrt{-1}(\alpha(s))\right) \\
\exp \left(+\frac{\pi}{2} \sqrt{-1}(\alpha(s))\right) & \exp \left(-\frac{\pi}{2} \sqrt{-1}(\alpha(s))\right)
\end{array}\right]} \\
& \quad \times\left[\begin{array}{cc}
\exp \left(-\frac{\pi}{4} \sqrt{-1}\left(\tau\left(\Lambda_{i-1}^{+}\right)-\tau\left(\Lambda_{i-1} \cap \Lambda_{i}\right)\right)\right) & 0 \\
0 & \exp \left(-\frac{\pi}{4} \sqrt{-1}\left(\tau\left(\Lambda_{i-1}^{-}\right)-\tau\left(\Lambda_{i-1} \cap \Lambda_{i}\right)\right)\right)
\end{array}\right] \\
& \quad \times\left[\begin{array}{l}
c_{i-1}^{+}(\vec{a}, s) \\
c_{i-1}^{-}(\vec{a}, s)
\end{array}\right]
\end{aligned}
$$

where

$$
\alpha(s)=\operatorname{order}_{\Lambda_{i-1}}-\operatorname{order}_{\Lambda_{i}}+\frac{1}{2}= \begin{cases}s+i & \text { when } \boldsymbol{V}=\mathrm{Mat}_{n} \mathbb{R} \\ s+2 i-1 & \text { when } \boldsymbol{V}=\operatorname{Alt}_{2 n} \mathbb{R}\end{cases}
$$

and

$$
\begin{aligned}
\tau\left(\Lambda_{i-1}^{\epsilon}\right) & =\operatorname{sgn}_{A \in \mathfrak{G}}\left(\left\langle A \cdot x_{i-1}^{\epsilon}, A^{*} \cdot y_{i-1}^{\epsilon}\right\rangle\right), \\
\tau\left(\Lambda_{i-1} \cap \Lambda_{i}\right) & =\operatorname{sgn}_{A \in \mathfrak{G}}\left(\left\langle A \cdot x_{0}, A^{*} \cdot y_{0}\right\rangle\right) .
\end{aligned}
$$

In this formula, $\operatorname{sgn}_{A \in \mathfrak{G}}(-)$ means the signature of the quadratic form on $A \in \mathfrak{G}$, i.e., the difference of the number of positive eigenvalues and that of negative eigenvalues of the symmetric matrix representing the quadratic form; $\left(x_{i-1}^{\epsilon}, y_{i-1}^{\epsilon}\right) \in \Lambda_{i-1}^{\epsilon}$ given by (28) and (29); and $\left(x_{0}, y_{0}\right)$ is the point given by (30) in the dense $\boldsymbol{G}$-orbit of $\Lambda_{i-1} \cap \Lambda_{i}$. We see easily that $\tau\left(\Lambda_{i-1}^{\epsilon}\right)=$ 0 and $\tau\left(\Lambda_{i-1} \cap \Lambda_{i}\right)=0$, then we have the formulas (43) and (44).

Thus, we can compute all the coefficients $c_{i}^{\epsilon}(\vec{a}, s)(0 \leq i \leq n$ and $\epsilon= \pm)$ from the base coefficients $c_{0}^{\epsilon}(\vec{a}, s)=a_{\epsilon}(\epsilon= \pm)$ by using the formula (43) and (44). By computing the exact orders of poles of all the coefficient functions in the set $\left\{c_{i}^{\epsilon}(\vec{a}, s) \mid 0 \leq i \leq n, \epsilon= \pm\right\}$, we can determine the exact orders of poles of $P^{[\vec{a}, s]}(x)$. Namely we have the following proposition, which will be used in Section 6 .

Proposition 5.3. The following two conditions are equivalent.

1. $P^{[\vec{a}, s]}(x)$ has a pole of order $p$ at $s=s_{0}$.

2. All the coefficient functions in $\left\{c_{i}^{\epsilon}(\vec{a}, s) \mid 0 \leq i \leq n, \epsilon= \pm\right\}$ have poles of order not greater than $p$ at $s=s_{0}$ and at least one coefficient of them has a pole of order $p$ at $s=s_{0}$. 
Proof. The proof is carried out in the same way as Muro [11, Proposition 3.7].

Next, we consider the Laurent expansion coefficients of the Laurent series expansion of $P^{[\vec{a}, s]}(x)$ at $s=s_{0}$. We can determine the exact support of the Laurent expansion coefficients by computing the orders of poles of the coefficient functions. Suppose that the complex power function $P^{[\vec{a}, s]}(x)$ has a pole of order $p$ at $s=s_{0}$. We give the Laurent expansion of $P^{[\vec{a}, s]}(x)$ at $s=s_{0}$ by

$$
P^{[\vec{a}, s]}(x)=\sum_{w=-p}^{\infty} P_{w}^{\left[\vec{a}, s_{0}\right]}(x)\left(s-s_{0}\right)^{w}
$$

Here, $P_{w}^{\left[\vec{a}, s_{0}\right]}(x)$ is called the Laurent expansion coefficient of degree $w$ of $P^{[\vec{a}, s]}(x)$. We can express the support of $P_{w}^{\left[\vec{a}, s_{0}\right]}(x)$ in terms of the order of poles of $c_{i}^{\epsilon}(\vec{a}, s)$. Namely, we have the following proposition, which will be used in Section 7.

Proposition 5.4. Suppose that $P^{[\vec{a}, s]}(x)$ has a pole of order $p$ at $s=$ $s_{0}$. Let $(47)$ be the Laurent expansion of $P^{[\vec{a}, s]}(x)$ at $s=s_{0}$ and let $c_{i}^{\epsilon}(\vec{a}, s)$ be the coefficient function defined by (41) and $\operatorname{ord}_{s=s_{0}}\left(c_{i}^{\epsilon}(\vec{a}, s)\right)$ stands for the order of pole of $c_{i}^{\epsilon}(\vec{a}, s)$ at $s=s_{0}$. Then, when $w<0$, we have

$$
\operatorname{Supp}\left(P_{w}^{\left[\vec{a}, s_{0}\right]}(x)\right)=\overline{\bigcup_{i \in L} \boldsymbol{S}_{i}}
$$

with $L:=\left\{i \in \mathbb{Z} \mid \operatorname{ord}_{s=s_{0}}\left(c_{i}^{\epsilon}(\vec{a}, s)\right) \geq-w\right.$ for $\epsilon=+$ or $\left.\epsilon=-\right\}$. When $w=$ 0 , we have

$$
\operatorname{Supp}\left(P_{0}^{\left[\vec{a}, s_{0}\right]}(x)\right)=\overline{\bigcup_{a_{\epsilon} \neq 0} \boldsymbol{V}_{\epsilon}}
$$

Proof. The proof is carried out in the same way as Muro [11, Proposition 3.8].

\section{§6. Exact orders of the poles of the complex powers}

In this section we give the answer to the first problem in Problem 1.1 in Section 1. We begin with the definitions about some special subspaces of $\mathbb{C}^{2}$ 
Definition 6.1. 1. Let $q \in \mathbb{Z}$. We define a vector subspace $A(\lambda, q)$ of $\mathbb{C}^{2}$ by

$$
A(\lambda, q):=\left\{\vec{a} \in \mathbb{C}^{2} \mid o(\vec{a}, \lambda) \leq q\right\}
$$

which consists of the vectors $\vec{a}$ such that $P^{[\vec{a}, s]}(x)$ has a pole of order at most $q$ at $s=\lambda$. Here $o(\vec{a}, \lambda)$ has been defined by $(23)$.

2. We define

$$
\begin{aligned}
& A_{\text {odd }}:=\left\{\vec{a} \in \mathbb{C}^{2} \mid a_{+}+a_{-}=0\right\} \\
& A_{\text {even }}:=\left\{\vec{a} \in \mathbb{C}^{2} \mid a_{+}-a_{-}=0\right\}
\end{aligned}
$$

Then $P^{[\vec{a}, s]}(x)$ is an odd (resp. even) function if $\vec{a} \in A_{\text {odd }}$ (resp. $\vec{a} \in A_{\text {even }}$ ).

Then the following proposition is easily verified from the definition.

Proposition 6.1. Let $A(\lambda, q)$ be the vector subspace of $\mathbb{C}^{2}$ defined by (50). Then $A(\lambda, q)$ has the following properties.

1. For any $q \in \mathbb{Z}$, we have

$$
A(\lambda, q) \subset A(\lambda, q+1)
$$

If $q \leq-1$, then $A(\lambda, q)=\{0\}$. If $q \geq \operatorname{PHO}(\lambda)$, then $A(\lambda, q)=\mathbb{C}^{2}$.

2. We define, for each $q \in \mathbb{Z}$,

$$
\overline{A(\lambda, q)}:=A(\lambda, q) / A(\lambda, q-1) .
$$

Then we have $\overline{A(\lambda, q)}=\{0\}$ if $q>P H O(\lambda)$ or $q<0$.

By determining the subspaces $A(\lambda, q)$ for each $\lambda \in \mathbb{C}$ and $q \in \mathbb{Z}$, we can compute the exact order of the pole of $P^{[\vec{a}, s]}(x)$ at $s=\lambda$. Namely, the exact order of the pole of $P^{[\vec{a}, s]}(x)$ at $s=\lambda$ is $q$ if and only if $\vec{a} \in A(\lambda, q)$ and the representative $[\vec{a}] \neq 0$ in $\overline{A(\lambda, q)}$.

If $\lambda \notin \mathbb{Z}_{<0}$, then $A(\lambda, q)$ is easily determined for each $q \in \mathbb{Z}$ since $P^{[\vec{a}, s]}(x)$ is holomorphic at $s=\lambda$ for all $\vec{a} \in \mathbb{C}^{2}$. Namely we have the following theorem.

TheOREM 6.2. Let $\boldsymbol{V}$ be $\operatorname{Mat}_{n}(\mathbb{R})$ or $\operatorname{Alt}_{2 n}(\mathbb{R})$. Suppose that $\lambda \notin \mathbb{Z}_{<0}$. Then we have

$$
A(\lambda, q)= \begin{cases}\mathbb{C}^{2} & \text { if } q \geq 0 \\ \{0\} & \text { if } q<0\end{cases}
$$


and hence

$$
\overline{A(\lambda, q)}= \begin{cases}\mathbb{C}^{2} & \text { if } q=0 \\ \{0\} & \text { if } q \neq 0\end{cases}
$$

for each $q \in \mathbb{Z}$.

For the square matrix space $\boldsymbol{V}=\operatorname{Mat}_{n}(\mathbb{R})$, we can determine $A(\lambda, q)$ for each $\lambda \in \mathbb{Z}_{<0}$ and $q \in \mathbb{Z}$ in the following theorem.

TheOREM 6.3. (square matrices) Let $\boldsymbol{V}$ be the space of $n \times n$ square matrices and let $\lambda$ be a negative integer.

1. We suppose that $-n+1 \leq \lambda \leq-1$. When $\lambda$ is an even integer,

$$
A(\lambda, q)= \begin{cases}\mathbb{C}^{2} & \text { if } q \geq-\frac{\lambda}{2}, \\ \{0\} & \text { if } q<-\frac{\lambda}{2},\end{cases}
$$

and hence

$$
\overline{A(\lambda, q)}= \begin{cases}\mathbb{C}^{2} & \text { if } q=-\frac{\lambda}{2}, \\ \{0\} & \text { if } q \neq-\frac{\lambda}{2} .\end{cases}
$$

When $\lambda$ is an odd integer,

$$
A(\lambda, q)= \begin{cases}\mathbb{C}^{2} & \text { if } q \geq \frac{-\lambda-1}{2}+1, \\ A_{\text {odd }} \simeq \mathbb{C} & \text { if } q=\frac{-\lambda-1}{2} \\ \{0\} & \text { if } q \leq \frac{-\lambda-1}{2}-1\end{cases}
$$

and hence

$$
\overline{A(\lambda, q)}= \begin{cases}\mathbb{C} & \text { if } q=\frac{-\lambda+1}{2}, \frac{-\lambda-1}{2}, \\ \{0\} & \text { if } q \neq \frac{-\lambda+1}{2}, \frac{-\lambda-1}{2} .\end{cases}
$$

2. We suppose that $\lambda \leq-n$. When $n$ is an even integer,

$$
A(\lambda, q)= \begin{cases}\mathbb{C}^{2} & \text { if } q \geq \frac{n}{2}, \\ \{0\} & \text { if } q<\frac{n}{2}\end{cases}
$$

and hence

$$
\overline{A(\lambda, q)}= \begin{cases}\mathbb{C}^{2} & \text { if } q=\frac{n}{2}, \\ \{0\} & \text { if } q \neq \frac{n}{2}\end{cases}
$$


When $n$ is an odd integer,

$$
A(\lambda, q)= \begin{cases}\mathbb{C}^{2} & \text { if } q \geq \frac{n-1}{2}+1 \\ A_{\text {even }} \simeq \mathbb{C} & \text { if } q=\frac{n-1}{2} \text { and } \lambda \text { is even } \\ A_{\text {odd }} \simeq \mathbb{C} & \text { if } q=\frac{n-1}{2} \text { and } \lambda \text { is odd } \\ \{0\} & \text { if } q \leq \frac{n-1}{2}-1\end{cases}
$$

and hence

$$
\overline{A(\lambda, q)}= \begin{cases}\mathbb{C} & \text { if } q=\frac{n+1}{2}, \frac{n-1}{2} \\ \{0\} & \text { if } q \neq \frac{n+1}{2}, \frac{n-1}{2}\end{cases}
$$

Proof. According to Proposition 5.3, the order of the pole of $P^{[\vec{a}, s]}(x)$ at $s=\lambda$ is determined by the maximum of the orders of poles of coefficient functions $c_{i}^{\epsilon}(\vec{a}, s)(0 \leq i \leq n$ and $\epsilon= \pm)$. By using the formula (43) repeatedly, we have

$$
\vec{c}_{m}(\vec{a}, s):=\left[\begin{array}{c}
c_{m}^{+}(\vec{a}, s) \\
c_{m}^{-}(\vec{a}, s)
\end{array}\right]=(2 \pi)^{-m / 2} \prod_{i=1}^{m} U_{i}(s) \vec{a}
$$

where

$$
U_{i}(s):=\Gamma(s+i) \times\left[\begin{array}{ll}
\exp \left(-\frac{\pi}{2} \sqrt{-1}(s+i)\right) & \exp \left(+\frac{\pi}{2} \sqrt{-1}(s+i)\right) \\
\exp \left(+\frac{\pi}{2} \sqrt{-1}(s+i)\right) & \exp \left(-\frac{\pi}{2} \sqrt{-1}(s+i)\right)
\end{array}\right]
$$

and $\vec{a}=\left(\begin{array}{c}a_{+} \\ a_{-}\end{array}\right)=\left[\begin{array}{l}c_{0}^{+}(\vec{a}, s) \\ c_{0}^{-}(\vec{a}, s)\end{array}\right] \in \mathbb{C}^{2}$. Then we can see easily that

$$
\begin{aligned}
(2 \pi)^{m / 2} \vec{c}_{m}(\overrightarrow{\text { even }}, s) & =\prod_{i=1}^{m} U_{i}(s)(\overrightarrow{\text { even }})=F_{m}(\overrightarrow{\text { even }}, s)(\overrightarrow{\text { even }}) \\
(2 \pi)^{m / 2} \vec{c}_{m}(\overrightarrow{\text { odd }}, s) & =\prod_{i=1}^{m} U_{i}(s)(\overrightarrow{\text { odd }})=F_{m}(\overrightarrow{\text { odd }}, s)(\overrightarrow{\text { odd }})
\end{aligned}
$$

where

$$
F_{m}(\overrightarrow{\mathrm{even}}, s)=\left\{\begin{array}{lr}
\prod_{i=1}^{m} \Gamma(s+i)(\sqrt{-1})^{k}\left(p^{-2}-p^{2}\right)^{k} & \text { when } m=2 k \\
\prod_{i=1}^{m} \Gamma(s+i)(-\sqrt{-1})^{k+1}\left(p^{-2}-p^{2}\right)^{k}\left(p^{-1}-p\right) & \text { when } m=2 k+1,
\end{array}\right.
$$


and

$$
F_{m}(\overrightarrow{\mathrm{odd}}, s)=\left\{\begin{array}{lr}
\prod_{i=1}^{m} \Gamma(s+i)(\sqrt{-1})^{k}\left(p^{-2}-p^{2}\right)^{k} & \text { when } m=2 k, \\
\prod_{i=1}^{m} \Gamma(s+i)(-\sqrt{-1})^{k+1}\left(p^{-2}-p^{2}\right)^{k}\left(p^{-1}+p\right) & \text { when } m=2 k+1,
\end{array}\right.
$$

with $p=\exp \left(\frac{\pi}{2} \sqrt{-1} s\right)$.

Then we are ready to determine the exact orders of the poles of $P^{[\vec{a}, s]}(x)$ at $s=\lambda$ in $\lambda \leq-1$.

1. We suppose that $-n+1 \leq \lambda \leq-1$.

First we assume that $\lambda$ is an even integer. Consider the orders of poles of $F_{m}(\overrightarrow{\text { even }}, s)$ and $F_{m}(\overrightarrow{\text { odd }}, s)(m=0,1, \ldots, n)$ at $s=\lambda$. Then the maximum of the orders of $F_{m}(\overrightarrow{\text { even }}, s)(m=0,1, \ldots, n)$ at $s=\lambda$ is $-\lambda / 2$, which is taken by $F_{-\lambda}(\overrightarrow{\text { even }}, s)$, and the maximum of the orders of $F_{m}(\overrightarrow{\text { odd }}, s)$ $(m=0,1, \ldots, n)$ at $s=\lambda$ is also $-\lambda / 2$, which is taken by $F_{-\lambda-1}(\overrightarrow{\mathrm{odd}}, s)$, $F_{-\lambda}(\overrightarrow{\text { odd }}, s)$ and $F_{-\lambda+1}(\overrightarrow{\text { odd }}, s)$. Let $\vec{a}:=a \cdot \overrightarrow{\text { even }}+b \cdot \overrightarrow{\text { odd }}$ with $a, b \in \mathbb{C}$. Then we have

$$
\vec{c}_{m}(\vec{a}, s)=a \cdot \vec{c}_{m}(\overrightarrow{\text { even }}, s)+b \cdot \vec{c}_{m}(\overrightarrow{\mathrm{odd}}, s)
$$

The vectors $\vec{c}_{m}(\overrightarrow{\text { even }}, s)$ and $\vec{c}_{m}(\overrightarrow{\text { odd }}, s)$ are linearly independent since they are constant multiples of $\overrightarrow{\text { even }}$ and $\overrightarrow{\mathrm{odd}}$, respectively. Consider the maximum of the orders of the poles of $\vec{c}_{m}(\vec{a}, s)(m=0,1, \ldots, n)$. If $\vec{a} \neq 0$, then it is the order of the pole of $\vec{c}_{-\lambda}(\vec{a}, s)=a \cdot \vec{c}_{-\lambda}(\overrightarrow{\text { even }}, s)+b \cdot \vec{c}_{-\lambda}(\overrightarrow{\text { odd }}, s)$, which is $-\lambda / 2$ by the above calculation. Then, by Proposition 5.3 , the order of the pole of $P^{[\vec{a}, s]}(x)$ at $s=\lambda$ is $-\lambda / 2$ if $\vec{a} \neq 0$. Therefore, we have

$$
o(\vec{a}, \lambda)= \begin{cases}-\lambda / 2 & \text { if } \vec{a} \neq 0 \\ -\infty & \text { if } \vec{a}=0\end{cases}
$$

and we obtain (53) and (54).

Next we assume that $\lambda$ is an odd integer. Consider the order of poles of $F_{m}(\overrightarrow{\text { even }}, s)$ and $F_{m}(\overrightarrow{\mathrm{odd}}, s)(m=0,1, \ldots, n)$ at $s=\lambda$. Then the maximum of the orders of $F_{m}(\overrightarrow{\text { even }}, s)(m=0,1, \ldots, n)$ at $s=\lambda$ is $-(\lambda-1) / 2$, which is taken by $F_{-\lambda}(\overrightarrow{\text { even }}, s)$, and the maximum of the orders of $F_{m}(\overrightarrow{\mathrm{odd}}, s)$ $(m=0,1, \ldots, n)$ at $s=\lambda$ is $-(\lambda+1) / 2$, which is taken by $F_{-\lambda-1}(\overrightarrow{\mathrm{odd}}, s)$, 
$F_{-\lambda}(\overrightarrow{\mathrm{odd}}, s)$ and $F_{-\lambda+1}(\overrightarrow{\mathrm{odd}}, s)$. We compute the maximum of the orders of the poles of $\vec{c}_{m}(\vec{a}, s)(m=0,1, \ldots, n)$. Note that $\vec{a}:=a \cdot \overrightarrow{\text { even }}+b \cdot \overrightarrow{\text { odd }}$ with $a, b \in \mathbb{C}$. If $a \neq 0$ in $\vec{a}=a \cdot \overrightarrow{\text { even }}+b \cdot \overrightarrow{\text { odd }}$, then it is the order of the pole of $\vec{c}_{-\lambda}(\vec{a}, s)=a \cdot \vec{c}_{-\lambda}(\overrightarrow{\text { even }}, s)+b \cdot \vec{c}_{-\lambda}(\overrightarrow{\text { odd }}, s)$, which is $-(\lambda-1) / 2$ from the above calculation. If $a=0$ and $b \neq 0$ in $\vec{a}=a \cdot \overrightarrow{\text { even }}+b \cdot \overrightarrow{\text { odd }}$, then it is the order of the pole of $\vec{c}_{-\lambda}(\vec{a}, s)=b \cdot \vec{c}_{-\lambda}(\overrightarrow{\mathrm{odd}}, s)$, which is $-(\lambda+1) / 2$. Then, by Proposition 5.3, the order of the pole of $P^{[\vec{a}, s]}(x)$ at $s=\lambda$ is $-(\lambda-1) / 2$. If $a \neq 0$ in $\vec{a}=a \cdot \overrightarrow{\text { even }}+b \cdot \overrightarrow{\mathrm{odd}}$, and it is $-(\lambda+1) / 2$ if $a=0$ and $b \neq 0$ in $\vec{a}=a \cdot \overrightarrow{\text { even }}+b \cdot \overrightarrow{\text { odd }}$, Therefore, we have

$$
o(\vec{a}, \lambda)= \begin{cases}-\frac{\lambda-1}{2} & \text { if } a \neq 0 \text { in } \vec{a}=a \cdot \overrightarrow{\mathrm{even}}+b \cdot \overrightarrow{\mathrm{odd}} \\ -\frac{\lambda+1}{2} & \text { if } a=0 \text { and } b \neq 0 \text { in } \vec{a}=a \cdot \overrightarrow{\mathrm{even}}+b \cdot \overrightarrow{\mathrm{odd}} \\ -\infty & \text { if } \vec{a}=0\end{cases}
$$

and we obtain (55), (56).

2. We suppose that $\lambda \leq-n$. Then we see easily that the the maximum of the orders of the poles of $\vec{c}_{m}(\vec{a}, s)(m=0,1, \ldots, n)$ is taken when $m=n$. In the same way as the proof of the case of $-n+1 \leq \lambda \leq-1$, we can prove (57) and (58) when $\lambda$ is an even integer and (59), (60) when $\lambda$ is an odd integer.

Next, we consider the exact order of $P^{[\vec{a}, s]}(x)$ on the alternating matrix space $\boldsymbol{V}=\operatorname{Alt}_{2 n}(\mathbb{R})$.

THEOREM 6.4. (alternating matrices) Let $\boldsymbol{V}$ be the space of $2 n \times 2 n$ alternating matrices and let $\lambda$ be a negative integer.

1. We suppose that $-2 n+2 \leq \lambda \leq-1$.

$$
A(\lambda, q)= \begin{cases}\mathbb{C}^{2} & \text { if } q \geq\left\lfloor\frac{-\lambda+1}{2}\right\rfloor, \\ A_{\text {even }} \simeq \mathbb{C} & \text { if }\left\lfloor\frac{-\lambda+1}{2}\right\rfloor-1 \geq q \geq 0 \text { and } \lambda \text { is even } \\ A_{\text {odd }} \simeq \mathbb{C} & \text { if }\left\lfloor\frac{-\lambda+1}{2}\right\rfloor-1 \geq q \geq 0 \text { and } \lambda \text { is odd } \\ \{0\} & \text { if }-1 \geq q\end{cases}
$$

and hence

$$
\overline{A(\lambda, q)}= \begin{cases}\mathbb{C} & \text { if } q=\left\lfloor\frac{-\lambda+1}{2}\right\rfloor \text { or } 0 \\ \{0\} & \text { if } q \neq\left\lfloor\frac{-\lambda+1}{2}\right\rfloor, 0\end{cases}
$$


where, for $q$ in $\left\lfloor\frac{-\lambda+1}{2}\right\rfloor-1 \geq q \geq 0$,

2. We suppose that $\lambda \leq-2 n+1$.

$$
A(\lambda, q)= \begin{cases}\mathbb{C}^{2} & \text { if } q \geq n, \\ A_{\text {even }} \simeq \mathbb{C} & \text { if } n-1 \geq q \geq 0 \text { and } \lambda \text { is even } \\ A_{\text {odd }} \simeq \mathbb{C} & \text { if } n-1 \geq q \geq 0 \text { and } \lambda \text { is odd } \\ \{0\} & \text { if }-1 \geq q,\end{cases}
$$

and hence

$$
\overline{A(\lambda, q)}= \begin{cases}\mathbb{C} & \text { if } q=n \text { or } 0 \\ \{0\} & \text { if } q \neq n, 0\end{cases}
$$

where, for $q$ in $n-1 \geq q \geq 0$,

Proof. We can prove this theorem in the same way as the proof of Proposition 6.3. By using the formula (44) repeatedly, we have

$$
\vec{c}_{m}(\vec{a}, s):=\left[\begin{array}{c}
c_{m}^{+}(\vec{a}, s) \\
c_{m}^{-}(\vec{a}, s)
\end{array}\right]=(2 \pi)^{-m / 2} \prod_{i=1}^{m} U_{i}(s) \vec{a}
$$

where

$$
\begin{aligned}
U_{i}(s):= & \Gamma(s+2 i-1)) \\
& \times\left[\begin{array}{ll}
\exp \left(-\frac{\pi}{2} \sqrt{-1}(s+2 i-1)\right) & \exp \left(+\frac{\pi}{2} \sqrt{-1}(s+2 i-1)\right) \\
\exp \left(+\frac{\pi}{2} \sqrt{-1}(s+2 i-1)\right) & \exp \left(-\frac{\pi}{2} \sqrt{-1}(s+2 i-1)\right)
\end{array}\right]
\end{aligned}
$$

and $\vec{a}=\left(\begin{array}{c}a_{+} \\ a_{-}\end{array}\right)=\left[\begin{array}{l}c_{0}^{+}(\vec{a}, s) \\ c_{0}^{-}(\vec{a}, s)\end{array}\right] \in \mathbb{C}^{2}$. Then we can easily see that

$$
\begin{aligned}
(2 \pi)^{m / 2} \vec{c}_{m}(\overrightarrow{\text { even }}, s) & =\prod_{i=1}^{m} U_{i}(s)(\overrightarrow{\text { even }})=F_{m}(\overrightarrow{\text { even }}, s)(\overrightarrow{\text { even }}), \\
(2 \pi)^{m / 2} \vec{c}_{m}(\overrightarrow{\text { odd }}, s) & =\prod_{i=1}^{m} U_{i}(s)(\overrightarrow{\text { odd }})=F_{m}(\overrightarrow{\text { odd }}, s)(\overrightarrow{\text { odd }}) .
\end{aligned}
$$


where

$$
\begin{aligned}
F_{m}(\overrightarrow{\text { even }}, s)= & \prod_{i=1}^{m} \Gamma(s+2 i-1) \times(-1)^{m(m+1) / 2} \\
& \times\left(\exp \left(-\frac{\pi}{2} \sqrt{-1}(s-1)\right)+\exp \left(\frac{\pi}{2} \sqrt{-1}(s-1)\right)\right)^{m}, \\
F_{m}(\overrightarrow{\mathrm{odd}}, s)= & \prod_{i=1}^{m} \Gamma(s+2 i-1) \times(-1)^{m(m+1) / 2} \\
& \times\left(\exp \left(-\frac{\pi}{2} \sqrt{-1}(s-1)\right)-\exp \left(\frac{\pi}{2} \sqrt{-1}(s-1)\right)\right)^{m} .
\end{aligned}
$$

We shall calculate the orders of the poles of $P^{[\vec{a}, s]}(x)$ at $s=\lambda$ with $\lambda \leq-1$.

1. We suppose that $-2 n+2 \leq \lambda \leq-1$. First we assume that $\lambda$ is an even integer. Consider the orders of poles of $F_{m}(\overrightarrow{\text { even }}, s)$ and $F_{m}(\overrightarrow{\text { odd }}, s)$ $(m=0,1, \ldots, n)$ at $s=\lambda$. Then all of $F_{m}(\overrightarrow{\text { even }}, s)(m=0,1, \ldots, n)$ is holomorphic at $s=\lambda$ and the order of $F_{m}(\overrightarrow{\mathrm{odd}}, s)$ at $s=\lambda$ is

$$
\begin{cases}m & \text { if } m \leq-\frac{\lambda}{2} \\ -\frac{\lambda}{2} & \text { if } m>-\frac{\lambda}{2}\end{cases}
$$

for $m=0,1, \ldots, n$. Let $\vec{a}:=a \cdot \overrightarrow{\text { even }}+b \cdot \overrightarrow{\text { odd }}$ with $a, b \in \mathbb{C}$. Then we have

$$
\vec{c}_{m}(\vec{a}, s)=a \cdot \vec{c}_{m}(\overrightarrow{\text { even }}, s)+b \cdot \vec{c}_{m}(\overrightarrow{\mathrm{odd}}, s) .
$$

The vectors $\vec{c}_{m}(\overrightarrow{\text { even }}, s)$ and $\vec{c}_{m}(\overrightarrow{\text { odd }}, s)$ are linearly independent for the same reason for the case of $\operatorname{Mat}_{n}(\mathbb{R})$. Consider the maximum of the orders of the poles of $\vec{c}_{m}(\vec{a}, s)(m=0,1, \ldots, n)$. If $\vec{a} \neq 0$ and $\vec{a} \notin A_{\text {even, }}$, then it is the order of the pole of $\vec{c}_{m}(\overrightarrow{\mathrm{odd}}, s)$ with $m=-\lambda / 2,-\lambda / 2+1, \ldots, n$, and the value is $-\lambda / 2$. Then, by Proposition 5.3, the order of the pole of $P^{[\vec{a}, s]}(x)$ at $s=\lambda$ is $-\lambda / 2$ if $\vec{a} \neq 0$ and $\vec{a} \notin A_{\text {even. }}$. If $\vec{a} \neq 0$ and $\vec{a} \in A_{\text {even }}$, then it is the order of the pole of $\vec{c}_{0}(\overrightarrow{\operatorname{even}}, s)$ and the value is 0 . Then, by Proposition 5.3, the order of the pole of $P^{[\vec{a}, s]}(x)$ at $s=\lambda$ is 0 if $\vec{a} \neq 0$ and $\vec{a} \in A_{\text {even. }}$. Therefore, we have

$$
o(\vec{a}, \lambda)= \begin{cases}-\frac{\lambda}{2} & \text { if } \vec{a} \neq 0 \text { and } \vec{a} \notin A_{\text {even }}, \\ 0 & \text { if } \vec{a} \neq 0 \text { and } \vec{a} \in A_{\text {even }} \\ -\infty & \text { if } \vec{a}=0\end{cases}
$$


when $\lambda$ is even.

Next we assume that $\lambda$ is an odd integer. Then all of $F_{m}(\overrightarrow{\text { odd }}, s)(m=$ $0,1, \ldots, n)$ is holomorphic at $s=\lambda$ and the order of $F_{m}(\overrightarrow{\text { even }}, s)$ at $s=\lambda$ is

$$
\begin{cases}m & \text { if } m \leq \frac{-\lambda+1}{2} \\ \frac{-\lambda+1}{2} & \text { if } m>\frac{-\lambda+1}{2}\end{cases}
$$

for $m=0,1, \ldots, n$. Consider the maximum of the orders of the poles of $\vec{c}_{m}(\vec{a}, s)(m=0,1, \ldots, n)$. If $\vec{a} \neq 0$ and $\vec{a} \notin A_{\text {odd }}$, then it is the order of the pole of $\vec{c}_{m}(\overrightarrow{\mathrm{even}}, s)$ with $m=(-\lambda+1) / 2,(-\lambda+1) / 2+1, \ldots, n$, and the value is $(-\lambda+1) / 2$. Then, by Proposition 5.3 , the order of the pole of $P^{[\vec{a}, s]}(x)$ at $s=\lambda$ is $(-\lambda+1) / 2$ if $\vec{a} \neq 0$ and $\vec{a} \notin A_{\text {odd }}$. If $\vec{a} \neq 0$ and $\vec{a} \in A_{\text {odd }}$, then it is the order of the pole of $\overrightarrow{c_{0}}(\overrightarrow{\mathrm{odd}}, s)$ and the value is 0 . Then, by Proposition 5.3, the order of the pole of $P^{[\vec{a}, s]}(x)$ at $s=\lambda$ is 0 if $\vec{a} \neq 0$ and $\vec{a} \in A_{\text {odd }}$. Therefore, we have

$$
o(\vec{a}, \lambda)= \begin{cases}\frac{-\lambda+1}{2} & \text { if } \vec{a} \neq 0 \text { and } \vec{a} \notin A_{\mathrm{odd}}, \\ 0 & \text { if } \vec{a} \neq 0 \text { and } \vec{a} \in A_{\mathrm{odd}} \\ -\infty & \text { if } \vec{a}=0\end{cases}
$$

when $\lambda$ is odd.

From (74) and (75), we obtain (66), (67) since

$$
\left\lfloor\frac{-\lambda+1}{2}\right\rfloor= \begin{cases}-\frac{\lambda}{2} & \text { if } \lambda \text { is even } \\ \frac{-\lambda+1}{2} & \text { if } \lambda \text { is odd }\end{cases}
$$

2. We suppose that $\lambda \leq-2 n+1$. Then we see easily that the the maximum of the orders of the poles of $\vec{c}_{m}(\vec{a}, s)(m=0,1, \ldots, n)$ is taken when $m=n$. In the same way as the proof of the case of $-2 n+2 \leq \lambda \leq-1$, we can prove (68), (69).

\section{$\S 7$. Exact support of Laurent expansion coefficients of complex powers}

In this section we give the answer to the second problem in Problem 1.1 in Section 1, i.e., the determination of the support of the Laurent expansion coefficients $P_{-q}^{[\vec{a}, \lambda]}(x)$ for each $q \in \mathbb{Z}_{>0}, \vec{a} \in \mathbb{C}^{2}$ and $\lambda \in \mathbb{C}$. For the square matrix space, we have the following exact support theorem on the Laurent expansion coefficients of $P^{[\vec{a}, s]}(x)$. 
TheOREM 7.1. (square matrices) Let $\boldsymbol{V}$ be the space of $n \times n$ square matrices and let $\lambda$ be a negative integer.

1. We suppose that $\lambda$ is an even integer. We put $m:=-\lambda / 2 \in \mathbb{Z}_{>0}$.

(a) Suppose that $\vec{a}:=\left(a_{+}, a_{-}\right) \notin A_{\text {even }}$. Then

$$
\operatorname{Supp}\left(P_{-q}^{[\vec{a}, \lambda]}(x)\right)= \begin{cases}\emptyset & \text { for } q=m+1, m+2, \ldots, \\ \overline{\boldsymbol{S}_{2 q-1}} & \text { for } q=1, \ldots, m \\ \overline{\bigcup_{a_{\epsilon} \neq 0} \boldsymbol{V}_{\epsilon}} & \text { for } q \leq 0\end{cases}
$$

if $m<\left\lfloor\frac{n+1}{2}\right\rfloor$ and

(77) $\operatorname{Supp}\left(P_{-q}^{[\vec{a}, \lambda]}(x)\right)= \begin{cases}\emptyset & \text { for } q=\left\lfloor\frac{n+1}{2}\right\rfloor+1,\left\lfloor\frac{n+1}{2}\right\rfloor+2, \ldots, \\ \overline{\boldsymbol{S}_{2 q-1}} & \text { for } q=1, \ldots,\left\lfloor\frac{n+1}{2}\right\rfloor, \\ \bigcup_{a_{\epsilon} \neq 0} \boldsymbol{V}_{\epsilon} & \text { for } q \leq 0,\end{cases}$ if $m \geq\left\lfloor\frac{n+1}{2}\right\rfloor$.

(b) Suppose that $\vec{a}:=\left(a_{+}, a_{-}\right) \in A_{\text {even }}$ and $\vec{a} \neq 0$. Then

(78) $\operatorname{Supp}\left(P_{-q}^{[\vec{a}, \lambda]}(x)\right)= \begin{cases}\emptyset & \text { for } q=m+1, m+2, \ldots, \\ \overline{\boldsymbol{S}_{2 q}} & \text { for } q=1, \ldots, m, \\ \bigcup_{a_{\epsilon} \neq 0} \boldsymbol{V}_{\epsilon}=\boldsymbol{V} & \text { for } q \leq 0,\end{cases}$ if $m<\left\lfloor\frac{n}{2}\right\rfloor$ and

$$
\operatorname{Supp}\left(P_{-q}^{[\vec{a}, \lambda]}(x)\right)= \begin{cases}\emptyset & \text { for } q=\left\lfloor\frac{n}{2}\right\rfloor+1,\left\lfloor\frac{n}{2}\right\rfloor+2, \ldots, \\ \overline{\boldsymbol{S}_{2 q}} & \text { for } q=1, \ldots,\left\lfloor\frac{n}{2}\right\rfloor, \\ \overline{\bigcup_{a_{\epsilon} \neq 0} \boldsymbol{V}_{\epsilon}}=\boldsymbol{V} & \text { for } q \leq 0,\end{cases}
$$

if $m \geq\left\lfloor\frac{n}{2}\right\rfloor$. $\mathbb{Z}_{>0}$

2. We suppose that $\lambda$ is an odd integer. We put $m:=(-\lambda+1) / 2 \in$

(a) Suppose that $\vec{a}:=\left(a_{+}, a_{-}\right) \notin A_{\text {odd }}$. Then

$$
\operatorname{Supp}\left(P_{-q}^{[\vec{a}, \lambda]}(x)\right)= \begin{cases}\emptyset & \text { for } q=m+1, m+2, \ldots, \\ \overline{\boldsymbol{S}_{2 q-1}} & \text { for } q=1, \ldots, m, \\ \overline{\bigcup_{a_{\epsilon} \neq 0} \boldsymbol{V}_{\epsilon}} & \text { for } q \leq 0,\end{cases}
$$


if $m<\left\lfloor\frac{n+1}{2}\right\rfloor$ and

(81) $\operatorname{Supp}\left(P_{-q}^{[\vec{a}, \lambda]}(x)\right)= \begin{cases}\emptyset & \text { for } q=\left\lfloor\frac{n+1}{2}\right\rfloor+1,\left\lfloor\frac{n+1}{2}\right\rfloor+2, \ldots, \\ \overline{\boldsymbol{S}_{2 q-1}} & \text { for } q=1, \ldots,\left\lfloor\frac{n+1}{2}\right\rfloor, \\ \overline{\bigcup_{a_{\epsilon} \neq 0} \boldsymbol{V}_{\epsilon}} & \text { for } q \leq 0,\end{cases}$ if $m \geq\left\lfloor\frac{n+1}{2}\right\rfloor$.

(b) Suppose that $\vec{a}:=\left(a_{+}, a_{-}\right) \in A_{\text {odd }}$ and $\vec{a} \neq 0$. Then

$$
\operatorname{Supp}\left(P_{-q}^{[\vec{a}, \lambda]}(x)\right)= \begin{cases}\emptyset & \text { for } q=m, m+1, \ldots, \\ \overline{\boldsymbol{S}_{2 q}} & \text { for } q=1, \ldots, m-1, \\ \overline{\bigcup_{a_{\epsilon} \neq 0} \boldsymbol{V}_{\epsilon}}=\boldsymbol{V} & \text { for } q \leq 0\end{cases}
$$

if $m-1<\left\lfloor\frac{n}{2}\right\rfloor$ and

$$
\operatorname{Supp}\left(P_{-q}^{[\vec{a}, \lambda]}(x)\right)= \begin{cases}\emptyset & \text { for } q=\left\lfloor\frac{n}{2}\right\rfloor+1,\left\lfloor\frac{n}{2}\right\rfloor+2, \ldots, \\ \overline{\boldsymbol{S}_{2 q}} & \text { for } q=1, \ldots,\left\lfloor\frac{n}{2}\right\rfloor, \\ \overline{\bigcup_{a_{\epsilon} \neq 0} \boldsymbol{V}_{\epsilon}}=\boldsymbol{V} & \text { for } q \leq 0,\end{cases}
$$

if $m-1 \geq\left\lfloor\frac{n}{2}\right\rfloor$.

Proof. First we suppose that $\lambda$ is a negative even integer and $\vec{a} \notin A_{\text {even }}$. Then the order of the pole of $P^{[\vec{a}, s]}(x)$ at $s=\lambda$ is

$$
\begin{cases}-\frac{\lambda}{2} & \text { if }-n+1 \leq \lambda \leq-1, \\ \left\lfloor\frac{n+1}{2}\right\rfloor & \text { if } \lambda \leq-n\end{cases}
$$

by Theorem 6.3. Consider the Laurent expansion of $P^{[\vec{a}, s]}(x)$ at $s=\lambda$

$$
P^{[\vec{a}, s]}(x)=\sum_{w \geq \max \left\{\frac{\lambda}{2},-\left\lfloor\frac{n+1}{2}\right\rfloor\right\}} P_{w}^{[\vec{a}, \lambda]}(x)(s-\lambda)^{-q} .
$$

From Proposition 5.4, when $w<0$, we have

$$
\operatorname{Supp}\left(P_{w}^{[\vec{a}, \lambda]}(x)\right)=\overline{\bigcup_{i \in L} \boldsymbol{S}_{i}}
$$

with $L:=\left\{i \in \mathbb{Z} \mid \operatorname{ord}_{s=s_{0}}\left(\vec{c}_{i}(\vec{a}, s)\right) \geq-w\right\}$ and when $w=0$, we have

$$
\operatorname{Supp}\left(P_{0}^{[\vec{a}, \lambda]}(x)\right)=\overline{\bigcup_{a_{\epsilon} \neq 0} \boldsymbol{V}_{\epsilon}}
$$


By using (64) and (65), only the orders of poles of $\vec{c}_{i}(\vec{a}, s)$ in $-\lambda-2 p-1 \leq$ $i \leq \min \{-\lambda+2 p+1, n\}$ are at least $q=-\frac{\lambda}{2}-p$. Here $p=0,1, \ldots,-\lambda / 2$. Therefore, if $q>0$, then we have

$$
\operatorname{Supp}\left(P_{-q}^{[\vec{a}, \lambda]}(x)\right)=\overline{\bigcup_{2 q-1 \leq i \leq \min \{-2 \lambda-2 q+1, n\}}} \boldsymbol{S}_{i}=\overline{\boldsymbol{S}_{2 q-1}}
$$

by applying the formula (85) since $-\lambda-2 p-1=2 q-1$ and $-\lambda+2 p+1=$ $-2 \lambda-2 q+1$. On the other hand, by the order formula of the pole (84), we have

$$
\operatorname{Supp}\left(P_{-q}^{[\vec{a}, \lambda]}(x)\right)=\emptyset \quad \text { if } q>-\frac{\lambda}{2}
$$

when $-n+1 \leq \lambda \leq-1$, and

$$
\operatorname{Supp}\left(P_{-q}^{[\vec{a}, \lambda]}(x)\right)=\emptyset \quad \text { if } q>\left\lfloor\frac{n+1}{2}\right\rfloor
$$

when $\lambda \leq-n$. Then we have (76) and (77) from the formula (86).

Next we suppose that $\lambda$ is a negative even integer and $\vec{a} \in A_{\text {even with }}$ $\vec{a} \neq 0$. Then the order of the pole of $P^{[\vec{a}, s]}(x)$ at $s=\lambda$ is

$$
\begin{cases}-\frac{\lambda}{2} & \text { if }-n+1 \leq \lambda \leq-1 \\ \left\lfloor\frac{n}{2}\right\rfloor & \text { if } \lambda \leq-n\end{cases}
$$

by Theorem 6.3. Consider the Laurent expansion of $P^{[\vec{a}, s]}(x)$ at $s=\lambda$

$$
P^{[\vec{a}, s]}(x)=\sum_{w \geq \max \left\{\frac{\lambda}{2},-\left\lfloor\frac{n}{2}\right\rfloor\right\}} P_{w}^{[\vec{a}, \lambda]}(x)(s-\lambda)^{-q} .
$$

By computing the orders of poles in (64) and (65), only the orders of poles of $\vec{c}_{i}(\vec{a}, s)$ in $-\lambda-2 p \leq i \leq \min \{-\lambda+2 p, n\}$ are at least $q=-\frac{\lambda}{2}-p$. Here $p=0,1, \ldots,-\lambda / 2$. Therefore, if $q>0$, then we have

$$
\operatorname{Supp}\left(P_{-q}^{[\vec{a}, \lambda]}(x)\right)=\overline{\bigcup_{2 q \leq i \leq \min \{-2 \lambda-2 q, n\}} \boldsymbol{S}_{i}}=\overline{\boldsymbol{S}_{2 q}}
$$

by applying the formula (85) since $-\lambda-2 p=2 q$ and $-\lambda+2 p=-2 \lambda-2 q$.

On the other hand, by the order formula of the pole (84), we have

$$
\operatorname{Supp}\left(P_{-q}^{[\vec{a}, \lambda]}(x)\right)=\emptyset \quad \text { if } q>-\frac{\lambda}{2}
$$


when $-n+1 \leq \lambda \leq-1$, and

$$
\operatorname{Supp}\left(P_{-q}^{[\vec{a}, \lambda]}(x)\right)=\emptyset \quad \text { if } q>\left\lfloor\frac{n+1}{2}\right\rfloor
$$

when $\lambda \leq-n$. Then we have (78) and (79) from the formula (86).

For the case that $\lambda$ is a negative odd integer, we can prove (80) and (81), (82) and (83) in the same way.

Next, we give the exact support theorem of the Laurent expansion coefficients of $P^{[\vec{a}, s]}(x)$ on the alternating matrix space.

THEOREM 7.2. (alternating matrices) Let $\boldsymbol{V}$ be the space of $2 n \times 2 n$ alternating matrices and let $\lambda$ be a negative integer.

1. We suppose that $\lambda$ is an even integer. We put $m:=-\lambda / 2 \in \mathbb{Z}_{>0}$.

(a) Suppose that $\vec{a}:=\left(a_{+}, a_{-}\right) \notin A_{\text {even }}$. Then

$$
\operatorname{Supp}\left(P_{-q}^{[\vec{a}, \lambda]}(x)\right)= \begin{cases}\emptyset & \text { for } q=m+1, m+2, \ldots, \\ \overline{\boldsymbol{S}_{q}} & \text { for } q=1, \ldots, m, \\ \overline{\bigcup_{a_{\epsilon} \neq 0} \boldsymbol{V}_{\epsilon}} & \text { for } q \leq 0,\end{cases}
$$

if $m<n$ and

$$
\operatorname{Supp}\left(P_{-q}^{[\vec{a}, \lambda]}(x)\right)= \begin{cases}\emptyset & \text { for } q=n+1, n+2, \ldots, \\ \overline{\boldsymbol{S}_{q}} & \text { for } q=1, \ldots, n, \\ \overline{\bigcup_{a_{\epsilon} \neq 0} \boldsymbol{V}_{\epsilon}} & \text { for } q \leq 0,\end{cases}
$$

if $m \geq n$.

(b) Suppose that $\vec{a}:=\left(a_{+}, a_{-}\right) \in A_{\text {even }}$ and $\vec{a} \neq 0$. Then

$$
\operatorname{Supp}\left(P_{-q}^{[\vec{a}, \lambda]}(x)\right)= \begin{cases}\emptyset & \text { for } q>0 \\ \overline{\bigcup_{a_{\epsilon} \neq 0} \boldsymbol{V}_{\epsilon}}=\boldsymbol{V} & \text { for } q \leq 0\end{cases}
$$

2. We suppose that $\lambda$ is an odd integer. We put $m:=\frac{-\lambda+1}{2} \in \mathbb{Z}_{>0}$.

(a) Suppose that $\vec{a}:=\left(a_{+}, a_{-}\right) \notin A_{\text {odd }}$. Then

$$
\operatorname{Supp}\left(P_{-q}^{[\vec{a}, \lambda]}(x)\right)= \begin{cases}\emptyset & \text { for } q=m+1, m+2, \ldots, \\ \overline{\boldsymbol{S}_{q}} & \text { for } q=1, \ldots, m, \\ \overline{\bigcup_{a_{\epsilon} \neq 0} \boldsymbol{V}_{\epsilon}} & \text { for } q \leq 0\end{cases}
$$


if $m<n$ and

$$
\operatorname{Supp}\left(P_{-q}^{[\vec{a}, \lambda]}(x)\right)= \begin{cases}\emptyset & \text { for } q=n+1, n+2, \ldots, \\ \overline{\boldsymbol{S}_{q}} & \text { for } q=1, \ldots, n, \\ \overline{\bigcup_{a_{\epsilon} \neq 0} \boldsymbol{V}_{\epsilon}} & \text { for } q \leq 0,\end{cases}
$$

if $m \geq n$.

(b) Suppose that $\vec{a}:=\left(a_{+}, a_{-}\right) \in A_{\text {odd }}$ and $\vec{a} \neq 0$. Then

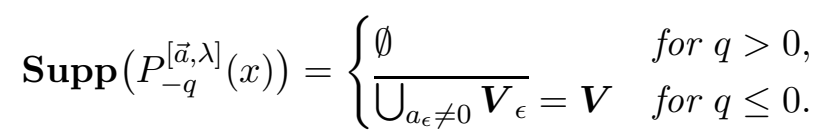

Proof. First we suppose that $\lambda$ is a negative even integer and $\vec{a} \notin A_{\text {even }}$. Then the order of the pole of $P^{[\vec{a}, s]}(x)$ at $s=\lambda$ is

$$
\begin{cases}-\frac{\lambda}{2} & \text { if }-2 n+1 \leq \lambda \leq-1 \\ n & \text { if } \lambda \leq-2 n\end{cases}
$$

by Theorem 6.4. (Note that $\left\lfloor\frac{-\lambda+1}{2}\right\rfloor=-\frac{\lambda}{2}$ when $\lambda$ is even.) Consider the Laurent expansion of $P^{[\vec{a}, s]}(x)$ at $s=\lambda$

$$
P^{[\vec{a}, s]}(x)=\sum_{w \geq \max \left\{\frac{\lambda}{2},-n\right\}} P_{w}^{[\vec{a}, \lambda]}(x)(s-\lambda)^{-q} .
$$

By computing the orders of poles in (64) and (65), only the orders of poles of $\vec{c}_{i}(\vec{a}, s)$ in $-\frac{\lambda}{2}-p \leq i \leq n$ are at least $q=-\frac{\lambda}{2}-p$. Here $p=0,1, \ldots,-\lambda / 2$. Therefore, if $q>0$, then we have

$$
\operatorname{Supp}\left(P_{-q}^{[\vec{a}, \lambda]}(x)\right)=\overline{\boldsymbol{S}_{q}}
$$

by applying the formula (48).

On the other hand, by the order formula of the pole (94), we have

$$
\operatorname{Supp}\left(P_{-q}^{[\vec{a}, \lambda]}(x)\right)=\emptyset \quad \text { if } q>-\frac{\lambda}{2}
$$

when $-2 n+1 \leq \lambda \leq-1$, and

$$
\operatorname{Supp}\left(P_{-q}^{[\vec{a}, \lambda]}(x)\right)=\emptyset \quad \text { if } q>n
$$

when $\lambda \leq-2 n$. Then we have (88) and (89) from the formula (49).

Next we suppose that $\lambda$ is a negative even integer and $\vec{a} \in A_{\text {even }}$ with $\vec{a} \neq 0$. Then $P^{[\vec{a}, s]}(x)$ is holomorphic with respect to $s$ at $s=\lambda$ and hence we have $(90)$.

For the case that $\lambda$ is a negative odd integer, we can prove (91), (92) and (93) in the same way. 


\section{§8. Application 1: Explicit description of quasi-relatively invari- ant hyperfunctions}

We have seen in Proposition 3.3 that a Laurent expansion coefficient of $P^{[\vec{a}, s]}(x)$ at $s=\lambda$ is a quasi- $\chi^{\lambda}$-invariant hyperfunction. In this section, we prove that every quasi- $\chi^{\lambda}$-invariant hyperfunction can be written as a linear combination of Laurent expansion coefficients of $P^{[\vec{a}, s]}(x)$ at $s=\lambda$ (Theorem 8.1). Furthermore, we see that every singular quasi- $\chi^{\lambda}$-invariant hyperfunction is in fact $\chi^{\lambda}$-invariant on the biggest-dimensional orbit of the support (Theorem 8.2).

THEOREM 8.1. Let $\lambda$ be a fixed complex number and let $Q I(\lambda, q)$ be the vector space of quasi- $\chi^{\lambda}$-invariant hyperfunctions whose quasi-relativedegrees are at most $q$. Then, the dimension of $Q I(\lambda, q)$ is $2(q+1)$ and the hyperfunctions

$$
\begin{aligned}
& P_{-o(\text { even }, \lambda)}^{[\overrightarrow{\mathrm{eve}}, \lambda]}(x), P_{-o(\overrightarrow{\mathrm{even}}, \lambda)+1}^{[\overrightarrow{\mathrm{even}}, \lambda]}(x), \ldots, P_{-o(\overrightarrow{\mathrm{even}}, \lambda)+q}^{[\overrightarrow{\mathrm{even}}, \lambda]}(x), \\
& P_{-o(\text { odd }, \lambda)}^{[\overrightarrow{\text { odd }}, \lambda]}(x), P_{-o(\text { odd }, \lambda)+1}^{[\overrightarrow{\text { odd }, \lambda]}}(x), \ldots, P_{-o(\text { odd }, \lambda)+q}^{[\overrightarrow{\text { odd }}, \lambda]}(x),
\end{aligned}
$$

form a basis of $Q I(\lambda, q)$.

Proof. We first prove that the elements in (95) are linearly independent. We suppose that

$$
f(x):=\sum_{i=0}^{q} a_{i} P_{-o(\overrightarrow{\mathrm{even}}, \lambda)+i}^{[\overrightarrow{\mathrm{even}}, \lambda]}(x)+b_{i} P_{-o(\overrightarrow{\mathrm{odd}}, \lambda)+i}^{[\overrightarrow{\mathrm{odd}}, \lambda]}(x)=0 .
$$

We shall prove that $a_{i}=0$ and $b_{i}=0$ for all $i=0, \ldots, q$ by induction on $q$. When $q=0$, we have

$$
P_{-o(\overrightarrow{\operatorname{even}}, \lambda)}^{[\overrightarrow{\mathrm{eve}}, \lambda]}(x) \quad \text { and } \quad P_{-o(\overrightarrow{\mathrm{odd}}, \lambda)}^{[\overrightarrow{\mathrm{odd}}, \lambda]}(x)
$$

are linearly independent. In fact, if $\lambda$ is not an negative integer, then $o(\overrightarrow{\text { even }}, \lambda)=o(\overrightarrow{\text { odd }}, \lambda)=0$, and hence $(97)$ are linearly independent since $P_{-o(\text { even }, \lambda)}^{[\overrightarrow{\mathrm{eve}}, \lambda]}(x)=P^{[\overrightarrow{\mathrm{even}}, \lambda]}(x)$ and $P_{-o(\overrightarrow{\mathrm{odd}}, \lambda)}^{[\overrightarrow{\mathrm{odd}}, \lambda]}(x)=P^{[\overrightarrow{\mathrm{odd}}, \lambda]}(x)$ from the definition. If $\lambda$ is an negative integer, then the supports of the hyperfunctions in (97) are different (Theorems 7.1 and 7.2), and hence (97) are linearly independent. Next, we assume that our assertion is valid when $q=k$ and 
suppose (96) for $q=k+1$. Let $\square:=\delta \chi\left(A_{0}\right)^{-1}\left(\left\langle A_{0} \cdot x, \partial\right\rangle-\lambda \delta \chi\left(A_{0}\right)\right)$ with $A_{0}$ being an element of $\mathfrak{G}$ satisfying $\delta \chi\left(A_{0}\right) \neq 0$. Then we have

$$
\square^{k+1} f(x)=a_{k+1} P_{-o(\overrightarrow{\text { even }}, \lambda)}^{[\overrightarrow{\mathrm{even}}, \lambda]}(x)+b_{k+1} P_{-o(\overrightarrow{\text { odd }}, \lambda)}^{[\overrightarrow{\mathrm{odd}}, \lambda]}(x)=0,
$$

by (25), and hence $a_{k+1}=b_{k+1}=0$. Then, from the induction hypothesis, we have $a_{i}=b_{i}=0$ for all $i=0, \ldots, k$, and, after all, we have $a_{i}=b_{i}=0$ for all $i=0, \ldots, k+1$. Thus our assertion is valid for $q=k+1$, and we have completed the proof of the linear independence of the hyperfunctions in (95) for all non-negative integer $q$.

The rest of the proof is devoted to showing that any element in $Q I(\lambda, q)$ is written as a linear combination of (95). We shall prove it by induction on $q$.

First we prove it when $q=0$. Then we have only to prove that any element of $Q I(\lambda, 0)$ is written as a linear sum of $P_{-o(\overrightarrow{\operatorname{even}}, \lambda)}^{[\overrightarrow{\mathrm{even}}, \lambda]}(x)$ and $P_{-o(\overrightarrow{\text { odd }}, \lambda)}^{[\overrightarrow{\mathrm{odd}}, \lambda]}(x)$. Since $\bigoplus_{q \in \mathbb{Z}} \overrightarrow{A(\lambda, q)} \simeq \mathbb{C}^{2}$, the following two cases occurs. (For the definition of $\overline{A(\lambda, q)}$, see Proposition 6.1.)

1. There is only one integer $q_{1}$ satisfying $\overline{A\left(\lambda, q_{1}\right)} \simeq \mathbb{C}^{2}$.

2. There are two integers $q_{1}, q_{2}$ with $q_{1}>q_{2}$ satisfying $\overline{A\left(\lambda, q_{1}\right)} \simeq$ $\overline{A\left(\lambda, q_{2}\right)} \simeq \mathbb{C}$.

In fact, the integers $q_{1}, q_{2}$ have been determined in Theorems $6.2,6.3$ and 6.4. Furthermore, it has been proved that

1. In the first case, $\overrightarrow{A\left(\lambda, q_{1}\right)}$ is generated by $\overrightarrow{\text { even }}$ and $\overrightarrow{\text { odd }}$.

2. In the second case, either one of the following two cases occur: $\overrightarrow{A\left(\lambda, q_{1}\right)}$ is generated by $\overrightarrow{\text { even }}$ and $\overrightarrow{A\left(\lambda, q_{2}\right)}$ is generated by $\overrightarrow{\mathrm{odd}}$, or, $\overrightarrow{A\left(\lambda, q_{1}\right)}$ is generated by $\overrightarrow{\text { odd }}$ and $\overrightarrow{A\left(\lambda, q_{2}\right)}$ is generated by $\overrightarrow{\text { even }}$.

Note that any vector $\vec{a} \in \mathbb{C}^{2}$ is written as $\vec{a}=a_{1} \cdot \overrightarrow{\text { even }}+a_{2} \cdot \overrightarrow{\text { odd }}$ with $a_{1}, a_{2} \in \mathbb{C}$.

First we consider the first case. By Proposition 4.1, any $\chi^{\lambda}$-invariant hyperfunction is written as a finite sum of some functions in $\left\{P_{-o(\vec{a}, \lambda)}^{[\vec{a}, \lambda]}(x) \mid\right.$ $\left.\vec{a} \in \mathbb{C}^{2}\right\}$. Since $o(\vec{a}, \lambda)=q_{1}$ if $\vec{a} \neq 0$ in this case, any $\chi^{\lambda}$-invariant hyperfunction is given by

$$
P_{-q_{1}}^{[\vec{a}, \lambda]}(x)=a_{1} P_{-q_{1}}^{[\overrightarrow{\mathrm{even}}, \lambda]}(x)+a_{2} P_{-q_{1}}^{[\overrightarrow{\mathrm{odd}}, \lambda]}(x)
$$

for some $\vec{a} \in \mathbb{C}^{2}$. This is what we want to prove. 
Next we consider the second case. We suppose first that $\overline{A\left(\lambda, q_{1}\right)}$ is generated by $\overrightarrow{\text { even }}$ and $\overrightarrow{A\left(\lambda, q_{2}\right)}$ is generated by $\overrightarrow{\text { odd }}$. Since $o(\vec{a}, \lambda)=q_{1}$ if $a_{1} \neq 0$ and $o(\vec{a}, \lambda)=q_{2}$ if $a_{1}=0, a_{2} \neq 0$, any $\chi^{\lambda}$-invariant hyperfunction is given as a linear combination of

$$
P_{-q_{1}}^{[\vec{a}, \lambda]}(x)=a_{1} P_{-q_{1}}^{[\overrightarrow{\mathrm{eve}}, \lambda]}(x)
$$

with $a_{1} \neq 0$, and

$$
P_{-q_{2}}^{[\vec{a}, \lambda]}(x)=a_{2} P_{-q_{2}}^{[\overrightarrow{\mathrm{dod}}, \lambda]}(x)
$$

with $a_{1}=0, a_{2} \neq 0$. This is what we want to prove. For the case when $\overrightarrow{A\left(\lambda, q_{1}\right)}$ is generated by $\overrightarrow{\text { odd }}$ and $\overrightarrow{A\left(\lambda, q_{2}\right)}$ is generated by $\overrightarrow{\text { even, we can }}$ carry out the proof in the same way. After all, we have proved that any $\chi^{\lambda}$-invariant hyperfunction is written as a linear sum of $P_{-o(\overrightarrow{\mathrm{even}}, \lambda)}^{[\overrightarrow{\mathrm{eve}}, \lambda]}(x)$ and $P_{-o(\text { odd }, \lambda)}^{[\overrightarrow{\text { odd }}, \lambda]}(x)$. Thus we have finished the proof for the case of $q=0$.

Next we shall prove the case of $q=k+1$ under the assumption that we have proved the case of $q=k$. We prove in the first case, i.e., $\overline{A\left(\lambda, q_{1}\right)} \simeq \mathbb{C}^{2}$. From the induction assumption, we see that

$$
\begin{aligned}
& P_{-q_{1}}^{[\overrightarrow{\mathrm{even}}, \lambda]}(x), P_{-q_{1}+1}^{[\overrightarrow{\mathrm{even}}, \lambda]}(x), \ldots, P_{-q_{1}+k}^{[\overrightarrow{\mathrm{even}}, \lambda]}(x), \\
& P_{-q_{1}}^{[\overrightarrow{\mathrm{dod}}, \lambda]}(x), P_{-q_{1}+1}^{[\overrightarrow{\mathrm{odd}}, \lambda]}(x), \ldots, P_{-q_{1}+k}^{[\overrightarrow{\mathrm{odd}}, \lambda]}(x),
\end{aligned}
$$

generate the vector space $Q I(\lambda, k)$. Let $f(x)$ be a quasi- $\chi^{\lambda}$-invariant hyperfunctions of quasi-relative-degree $k+1$. Then, from Definition 2.1,

$$
g(x):=\square f(x)
$$

is a quasi- $\chi^{\lambda}$-invariant hyperfunctions of quasi-relative-degree $k$ and hence it is given as a linear combination of the hyperfunctions in (98):

$$
g(x)=\sum_{i=0}^{k} a_{i+1} P_{-q_{1}+i}^{[\overrightarrow{\mathrm{even}}, \lambda]}(x)+b_{i+1} P_{-q_{1}+i}^{[\overrightarrow{\mathrm{odd}}, \lambda]}(x)
$$

with $a_{i+1}, b_{i+1} \in \mathbb{C}(i=0, \ldots, k)$. Then, by putting

$$
\widetilde{g}(x):=\sum_{i=0}^{k} a_{i+1} P_{-q_{1}+i+1}^{[\overrightarrow{\mathrm{even}}, \lambda]}(x)+b_{i+1} P_{-q_{1}+i+1}^{[\overrightarrow{\mathrm{ddd}}, \lambda]}(x),
$$


we have

$$
\square(f(x)-\widetilde{g}(x))=0
$$

from (25). Then, since $f(x)-\widetilde{g}(x)$ is quasi- $\chi^{\lambda}$-invariant and hence $\boldsymbol{G}^{1}$ invariant by Proposition 2.1, we have $f(x)-\widetilde{g}(x) \in Q I(\lambda, 0)$ by Proposition 2.1. Therefore, from the proof of the case when $q=0, f(x)-\widetilde{g}(x)$ is written as

$$
f(x)-\widetilde{g}(x)=a_{0} P_{-q_{1}}^{[\overrightarrow{\mathrm{even}}, \lambda]}(x)+b_{0} P_{-q_{1}}^{[\overrightarrow{\mathrm{odd}}, \lambda]}(x),
$$

with $a_{0}, b_{0} \in \mathbb{C}$. Then we have

$$
f(x)=a_{0} P_{-q_{1}}^{[\overrightarrow{\mathrm{even}}, \lambda]}(x)+b_{0} P_{-q_{1}}^{[\overrightarrow{\mathrm{odd}}, \lambda]}(x)+\widetilde{g}(x)
$$

and hence $f(x)$ is written as a linear combination of

$$
\begin{aligned}
& P_{-q_{1}}^{[\overrightarrow{\mathrm{even}}, \lambda]}(x), P_{-q_{1}+1}^{[\overrightarrow{\mathrm{even}}, \lambda]}(x), \ldots, P_{-q_{1}+k+1}^{[\overrightarrow{\mathrm{even}}, \lambda]}(x), \\
& P_{-q_{1}}^{[\overrightarrow{\mathrm{odd}}, \lambda]}(x), P_{-q_{1}+1}^{[\overrightarrow{\mathrm{odd}}, \lambda]}(x), \ldots, P_{-q_{1}+k+1}^{[\overrightarrow{\mathrm{odd}}, \lambda]}(x) .
\end{aligned}
$$

This is the proof of the induction step for the first case. For the second case, i.e., $\overline{A\left(\lambda, q_{1}\right)} \simeq \overline{A\left(\lambda, q_{2}\right)} \simeq \mathbb{C}$, we can prove the induction step of the proof in the same way.

After all, we can prove that if any element of $Q I(\lambda, k)$ is written as a linear combination of

$$
\begin{aligned}
& P_{-o(\overrightarrow{\text { even }}, \lambda)}^{[\overrightarrow{\operatorname{even}}, \lambda]}(x), P_{-o(\overrightarrow{\text { even }}, \lambda)+1}^{[\overrightarrow{\text { even }, \lambda]}}(x), \ldots, P_{-o(\overrightarrow{\text { even }}, \lambda)+k}^{[\overrightarrow{\text { even }, \lambda]}}(x), \\
& P_{-o(\overrightarrow{\text { odd }}, \lambda)}^{[\overrightarrow{\text { odd }}, \lambda]}(x), P_{-o(\overrightarrow{\text { odd }}, \lambda)+1}^{[\overrightarrow{\text { odd }}, \lambda]}(x), \ldots, P_{-o(\overrightarrow{\text { odd }}, \lambda)+k}^{[\overrightarrow{\text { odd }}, \lambda]}
\end{aligned}
$$

then any element of $Q I(\lambda, k+1)$ is written as a linear combination of

$$
\begin{aligned}
& P_{-o(\overrightarrow{\text { even }}, \lambda)}^{[\overrightarrow{\text { even }, \lambda]}}(x), P_{-o(\overrightarrow{\text { even }}, \lambda)+1}^{[\overrightarrow{\text { even }, \lambda]}}(x), \ldots, P_{-o(\overrightarrow{\text { even }}, \lambda)+k+1}^{[\overrightarrow{\text { even }, \lambda]}}(x), \\
& P_{-o(\overrightarrow{\text { odd }}, \lambda)}^{[\overrightarrow{\text { odd }, \lambda]}}(x), P_{-o(\overrightarrow{\text { odd }}, \lambda)+1}^{[\overrightarrow{\text { odd }}, \lambda]}(x), \ldots, P_{-o(\overrightarrow{\text { odd }}, \lambda)+k+1}^{[\overrightarrow{\text { odd }}, \lambda]}(x) .
\end{aligned}
$$

Thus the proof is completed by induction on $q$.

As an application of Theorem 8.1, we have the following theorem. This theorem asserts that any singular quasi- $\chi^{\lambda}$-invariant hyperfunction is almost $\chi^{\lambda}$-invariant. 
THEOREM 8.2. Let $f(x)$ be a quasi- $\chi^{\lambda}$-invariant hyperfunction and we suppose that $f(s)$ is singular, i.e., the support of $f(x)$ is contained in the singular set $\boldsymbol{S}:=\{x \in \boldsymbol{V} \mid \operatorname{det}(x)=0\}$. Then there exists an integer $i_{0}>0$ satisfying $\boldsymbol{\operatorname { S u p p }}(f(x))=\overline{\boldsymbol{S}_{i_{0}}}$. Furthermore, for any point $x_{0} \in \boldsymbol{S}_{i_{0}}$, there exists a neighborhood $U_{x_{0}}$ of $x_{0}$ such that $f(x)$ is $\chi^{\lambda}$-invariant on $U_{x_{0}}$.

Proof. In Theorem 8.1, we have seen that any quasi- $\chi^{\lambda}$-invariant hyperfunction $f(x)$ is written as a linear combination of Laurent expansion coefficients at $s=\lambda$ of $P^{[\overrightarrow{\text { even }}, s]}(x)$ and $P^{[\overrightarrow{\text { odd }}, s]}(x)$. By Theorem 8.1, if $f(x)$ is singular, it is written as

$$
f(x)=\sum_{p<0} a_{p} P_{p}^{[\overrightarrow{\text { even }}, \lambda]}(x)+\sum_{q<0} b_{q} P_{q}^{[\overrightarrow{\text { odd }}, \lambda]}(x)
$$

with negative integers $p, q$ and complex coefficients $a_{p}, b_{q} \in \mathbb{C}$. By Theorem 7.1 or Theorem 7.2 , the support of each $P_{p}^{[\overrightarrow{\mathrm{even}}, \lambda]}(x)$ or $P_{q}^{[\overrightarrow{\mathrm{odd}}, \lambda]}(x)$ is one of the closures $\overline{\boldsymbol{S}_{i}}$ of the singular orbits $\boldsymbol{S}_{i}(i=1, \ldots, n)$. Let $\overline{\boldsymbol{S}_{i_{0}}}$ be the biggest-dimensional orbit appearing there and let $A_{0} \in \mathfrak{G}$ be an element satisfying $\delta \chi\left(A_{0}\right) \neq 0$. Then we have

$$
\begin{aligned}
g(x) & =\left(\delta \chi\left(A_{0}\right)\right)^{-1}\left(\left\langle A_{0} \cdot x, \partial\right\rangle-\lambda \delta \chi\left(A_{0}\right)\right) f(x) \\
& =\sum_{p<0} a_{p} P_{p-1}^{[\overrightarrow{\mathrm{even}}, \lambda]}(x)+\sum_{q<0} b_{q} P_{q-1}^{[\overrightarrow{\mathrm{odd}}, \lambda]}(x)
\end{aligned}
$$

by $(25)$. The supports of $P_{p-1}^{[\overrightarrow{\mathrm{even}}, \lambda]}(x)$ and $P_{q-1}^{[\overrightarrow{\mathrm{odd}}, \lambda]}(x)$ appearing in $(100)$ are contained in $\overline{\boldsymbol{S}_{i_{0}+1}}$ since the supports of those appearing in (99) are contained in $\overline{\boldsymbol{S}_{i_{0}}}$. Therefore we have $\operatorname{Supp}(g(x)) \subset \overline{\boldsymbol{S}_{i_{0}+1}}$. Then, for any point $x_{0} \in \boldsymbol{S}_{i_{0}}=\overline{\boldsymbol{S}_{i_{0}}}-\overline{\boldsymbol{S}_{i_{0}+1}}$, there exists a neighborhood $U_{x_{0}}$ satisfying $U_{x_{0}} \cap \overline{\boldsymbol{S}_{i_{0}+1}}=\emptyset$ and $g(x)=0$ on $U_{x_{0}}$. This means that $f(x)$ is $\chi^{\lambda}$-invariant on $U_{x_{0}}$.

If $f(x)$ is a quasi- $\chi^{\lambda}$-invariant hyperfunction with support on the open orbits, then it contains the terms of the form

$$
a_{+}|P(x)|_{+}^{\lambda}(\log |P(x)|)^{k}+a_{-}|P(x)|_{-}^{\lambda}(\log |P(x)|)^{k}
$$

with $k \in \mathbb{Z}_{\geq 0}$. Then $f(x)$ is quasi- $\chi^{\lambda}$-invariant on $\boldsymbol{V}-\boldsymbol{S}$, but not $\chi^{\lambda}$ invariant on $\boldsymbol{V}-\boldsymbol{S}$ though the open orbit is the largest-dimensional orbit in the support. On the other hand, Theorem 8.2 shows that $f(x)$ is in fact $\chi^{\lambda}$-invariant on the largest-dimensional orbit in the support if the support of $f(x)$ is contained in $\boldsymbol{S}$. Such phenomenon can be observed in some other prehomogeneous vector spaces. 


\section{§9. Application 2: Fourier transforms of singular $G^{1}$-invariant tempered distributions}

The second application is the computation of the Fourier transform of singular $\boldsymbol{G}^{1}$-invariant tempered distributions. We have seen in Proposition 4.2 that any singular $\boldsymbol{G}^{1}$-invariant tempered distribution is written as a linear combination of Laurent expansion coefficients of $P^{[\vec{a}, s]}(x)$ of negative degree at $s=-1,-2, \ldots$ Then we have only to compute the Fourier transform of the Laurent expansion coefficients of negative degree for the computation of the Fourier transforms of singular $\boldsymbol{G}^{1}$-invariant tempered distributions. By using the result in Sections 6 and 7, we can see what singular Laurent expansion coefficients come out from the Laurent expansions of $P^{[\vec{a}, s]}(x)$. In this section, we give the formulas of the Fourier transforms of each singular Laurent expansion coefficients (Theorems 9.3, 9.4 for the square matrix spaces and Theorems 9.7, 9.8 for the alternating matrix spaces). In Corollarys 9.5, 9.6 and 9.9, we give some results on bi-singular tempered distributions.

We begin with the computation of the Fourier transform of the relatively invariant tempered distribution $P^{[\vec{a}, s]}(x)$ with a meromorphic parameter $s \in \mathbb{C}$. Though the result of the calculation of the Fourier transform of $P^{[\vec{a}, s]}(x)$ is well known, we give here the same result by using the micro-local method.

In this paper, the Fourier transform of a tempered distribution $f(x)$ is denoted by

$$
f(x)^{\vee}=f^{\vee}(y)=\int f(x) \exp (-\sqrt{-1}\langle x, y\rangle) d x
$$

All the hyperfunctions in this section are tempered distributions and the Fourier transforms are treated in the framework of the Schwartz's theory of Fourier transforms of tempered distributions.

Proposition 9.1. The Fourier transforms of $|P(x)|_{\text {even }}^{s}$ and $|P(x)|_{\text {odd }}^{s}$ are given by the following formulas.

1. (The case of square matrices) 
(a) When $n=2 k$, we have

$$
\begin{aligned}
\int & \left\{\begin{array}{l}
|P(x)|_{\text {even }}^{s} \\
|P(x)|_{\text {odd }}^{\text {od }}
\end{array}\right\} \exp (-\sqrt{-1}\langle x, y\rangle) d x \\
= & (2 \pi)^{n^{2} / 2-n / 2} \times \Gamma(s+1) \Gamma(s+2) \cdots \Gamma(s+n) \\
& \times(\sqrt{-1})^{k}(\exp (-\pi \sqrt{-1} s)-\exp (\pi \sqrt{-1} s))^{k}\left\{\begin{array}{l}
|P(y)|_{\text {even }}^{-s-n} \\
|P(y)|_{\text {odd }}^{-s-n}
\end{array}\right\}
\end{aligned}
$$

(b) When $n=2 k+1$, we have

$$
\begin{aligned}
& \int\left\{\begin{array}{l}
|P(x)|_{\text {even }}^{s} \\
|P(x)|_{\text {odd }}^{s}
\end{array}\right\} \exp (-\sqrt{-1}\langle x, y\rangle) d x \\
& =(2 \pi)^{n^{2} / 2-n / 2} \times \Gamma(s+1) \Gamma(s+2) \cdots \Gamma(s+n) \\
& \times(-\sqrt{-1})^{k+1}(\exp (-\pi \sqrt{-1} s)-\exp (\pi \sqrt{-1} s))^{k} \\
& \times\left\{\begin{array}{l}
(\exp (-\pi \sqrt{-1} s / 2)-\exp (\pi \sqrt{-1} s / 2))|P(y)|_{\text {even }}^{-s-n} \\
(\exp (-\pi \sqrt{-1} s / 2)+\exp (\pi \sqrt{-1} s / 2))|P(y)|_{\text {odd }}^{-s-n}
\end{array}\right\}
\end{aligned}
$$

2. (The case of alternating matrices)

$$
\begin{aligned}
& \left.\int \begin{array}{l}
|P(x)|_{\text {even }}^{s} \\
|P(x)|_{\text {odd }}^{s}
\end{array}\right\} \exp (-\sqrt{-1}\langle x, y\rangle) d x \\
& =(2 \pi)^{n(n-1)} \cdot(-1)^{n(n+1) / 2} \cdot \Gamma(s+1) \Gamma(s+3) \cdots \Gamma(s+2 n-1) \\
& \quad \times(\sqrt{-1})^{n} \times\left\{\begin{array}{l}
(\exp (-\pi \sqrt{-1} s / 2)-\exp (\pi \sqrt{-1} s / 2))^{n}|P(y)|_{\text {even }}^{-s-2 n+1} \\
(\exp (-\pi \sqrt{-1} s / 2)+\exp (\pi \sqrt{-1} s / 2))^{n}|P(y)|_{\text {odd }}^{-s-2 n+1}
\end{array}\right\}
\end{aligned}
$$

Proof. We give an outline of the computation of the Fourier transforms by using micro-local calculus. The following lemma is a direct consequence of the general formula of the Fourier transform of a homogeneous regular holonomic tempered distribution by the micro-local method. This means that the principal symbol of the homogeneous regular holonomic tempered distribution on the conormal bundle is written by its Fourier transform. The proof was given by Kashiwara-Miwa [7, Lemma 2.2 in p. 123], and we do not give the proof here. 
LEMma 9.2. Note that, by (41), the principal symbol of $P^{[\vec{a}, s]}(x)$ on the conormal bundle of the origin $\Lambda_{n}=\{0\} \times \boldsymbol{V}^{*} \subset T^{*} \boldsymbol{V}$ is given by

$$
\sigma_{\Lambda_{n}^{\epsilon}}\left(P^{[\vec{a}, s]}(x)\right)=c_{n}^{\epsilon}(\vec{a}, s) \Omega_{i}^{\epsilon}(s) / \sqrt{|d x|} .
$$

Then we have

1. (the case of square matrices)

$$
\int P^{[\vec{a}, s]}(x) \exp (-\sqrt{-1}\langle x, y\rangle) d x=(2 \pi)^{n^{2} / 2} \sum_{\epsilon= \pm} c_{n}^{\epsilon}(\vec{a}, s)|P(y)|_{\epsilon}^{-s-n}
$$

2. (the case of alternating matrices)

$$
\begin{aligned}
\int & P^{[\vec{a}, s]}(x) \exp (-\sqrt{-1}\langle x, y\rangle) d x \\
= & (2 \pi)^{n(2 n-1) / 2} \sum_{\epsilon= \pm} c_{n}^{\epsilon}(\vec{a}, s)|P(y)|_{\epsilon}^{-s-2 n+1} .
\end{aligned}
$$

We have already calculated $\vec{c}_{n}(\vec{a}, s)=\left[\begin{array}{c}c_{n}^{+}(\vec{a}, s) \\ c_{n}^{-}(\vec{a}, s)\end{array}\right]$ by $(61)$ in the proof of Theorem 6.3 in the case of square matrix space and by (70) in the proof of Theorem 6.4 in the case of alternating matrix space.

We consider the case of square matrix space. By (63), we have

$$
\begin{aligned}
& \int P^{[\overrightarrow{\mathrm{even}}, s]}(x) \exp (-\sqrt{-1}\langle x, y\rangle) d x=\int|P(x)|_{\text {even }}^{s} \exp (-\sqrt{-1}\langle x, y\rangle) d x \\
& =(2 \pi)^{n^{2} / 2}\left(c_{n}^{+}(\overrightarrow{\mathrm{even}}, s)|P(y)|_{+}^{-s-n}+c_{n}^{-}(\overrightarrow{\mathrm{even}}, s)|P(y)|_{-}^{-s-n}\right) \\
& =(2 \pi)^{n^{2} / 2-n / 2} F_{n}(\overrightarrow{\mathrm{even}}, s)|P(y)|_{\text {even }}^{-s-n}
\end{aligned}
$$

where $F_{n}(\overrightarrow{\text { even }}, s)$ is defined in (64). Similarly, we also have

$$
\int|P(x)|_{\text {odd }}^{s} \exp (-\sqrt{-1}\langle x, y\rangle) d x=(2 \pi)^{n^{2} / 2-n / 2} F_{n}(\overrightarrow{\text { odd }}, s)|P(y)|_{\text {odd }}^{-s-n}
$$

where $F_{n}(\overrightarrow{\mathrm{odd}}, s)$ is defined in $(65)$. From the explicit computation of $F_{n}(\overrightarrow{\text { even }}, s)$ and $F_{n}(\overrightarrow{\text { odd }}, s)$ in the proof of Theorem 6.3 , we have the formulas of Fourier transform (101) and (102).

For the case of alternating matrix space, we have the formula of Fourier transform (103) by using (70), (72) in the proof of Theorem 6.4 and the explicit forms of $F_{n}(\overrightarrow{\text { even }}, s)$ and $F_{n}(\overrightarrow{\text { odd }}, s)$ in $(73)$. 
Now we go to the formulas of the Fourier transforms of the singular Laurent expansion coefficients. First we consider the square matrix space. Let $\boldsymbol{V}$ be the space of $n \times n$ real square matrices. We set

$$
\begin{aligned}
\left\{\begin{array}{l}
A_{\text {odd }}(s) \\
A_{\text {even }}(s)
\end{array}\right\}:= & (2 \pi)^{n^{2} / 2-n / 2} \times \Gamma(s+1) \Gamma(s+2) \cdots \Gamma(s+n) \\
& \times(\sqrt{-1})^{n / 2}(\exp (-\pi \sqrt{-1} s)-\exp (\pi \sqrt{-1} s))^{n / 2}
\end{aligned}
$$

if $n$ is an even integer, and

$$
\begin{aligned}
\left\{\begin{array}{c}
A_{\text {odd }}(s) \\
A_{\text {even }}(s)
\end{array}\right\}:= & (2 \pi)^{n^{2} / 2-n / 2} \times \Gamma(s+1) \Gamma(s+2) \cdots \Gamma(s+n) \\
& \times(-\sqrt{-1})^{(n-1) / 2+1}(\exp (-\pi \sqrt{-1} s)-\exp (\pi \sqrt{-1} s))^{(n-1) / 2} \\
& \times\left\{\begin{array}{c}
(\exp (-\pi \sqrt{-1} s / 2)+\exp (\pi \sqrt{-1} s / 2)) \\
(\exp (-\pi \sqrt{-1} s / 2)-\exp (\pi \sqrt{-1} s / 2))
\end{array}\right\}
\end{aligned}
$$

if $n$ is an odd integer. They are the coefficients of the formulas of the Fourier transforms in (101) and (102). We denote by $A_{\text {odd }}(\lambda)_{l}\left(\right.$ resp. $\left.A_{\text {even }}(\lambda)_{l}\right)$ the coefficients of the Laurent series expansions $A_{\text {odd }}(s)=\sum_{l \in \mathbb{Z}} A_{\text {odd }}(\lambda)_{l}(s-\lambda)^{l}$ $\left(\operatorname{resp} . A_{\text {even }}(s)=\sum_{l \in \mathbb{Z}} A_{\text {even }}(\lambda)_{l}(s-\lambda)^{l}\right)$. When $-n+1 \leq \lambda \leq-1$, it is easily seen that $A_{\text {odd }}(s)$ (resp. $A_{\text {even }}(s)$ ) has a pole of order $-l_{\text {odd }}$ (resp. $\left.-l_{\text {even }}\right)$ at $s=\lambda$ where

$$
\begin{gathered}
l_{\text {odd }}:= \begin{cases}\left\lfloor\frac{n}{2}\right\rfloor+\lambda & \text { if } \lambda \text { is an even integer, } \\
\left\lfloor\frac{n+1}{2}\right\rfloor+\lambda & \text { if } \lambda \text { is an odd integer. }\end{cases} \\
\text { (resp. } l_{\text {even }}:=\left\{\begin{array}{ll}
\left\lfloor\frac{n+1}{2}\right\rfloor+\lambda & \text { if } \lambda \text { is an even integer, } \\
\left\lfloor\frac{n}{2}\right\rfloor+\lambda & \text { if } \lambda \text { is an odd integer. }
\end{array}\right)
\end{gathered}
$$

If $\lambda \leq-n$, then $A_{\text {odd }}(s)$ (resp. $A_{\text {even }}(s)$ ) has a pole of order $-l_{\text {odd }}$ (resp. $\left.-l_{\text {even }}\right)$ at $s=\lambda$ where

$$
\begin{gathered}
l_{\text {odd }}:= \begin{cases}-n / 2 & \text { when } n \text { is even, } \\
-(n+1) / 2 & \text { when } n \text { is odd and } \lambda \text { is even, } \\
-(n-1) / 2 & \text { when } n \text { is odd and } \lambda \text { is odd, }\end{cases} \\
\left(\text { resp. } l_{\text {even }}:=\left\{\begin{array}{ll}
-n / 2 & \text { when } n \text { is even, } \\
-(n+1) / 2 & \text { when } n \text { is odd and } \lambda \text { is odd, } \\
-(n-1) / 2 & \text { when } n \text { is odd and } \lambda \text { is even. }
\end{array}\right)\right.
\end{gathered}
$$


Theorem 9.3. (square matrices 1) Let $\boldsymbol{V}$ be the space of $n \times n$ real square matrices and let $\lambda$ be an integer in $-n+1 \leq \lambda \leq-1$. The functions $A_{\text {even }}(s)$ and $A_{\text {odd }}(s)$ are defined by (107) and (108). Then we have:

1. Suppose that $\lambda$ is even. We set $m:=-\lambda / 2$. Then we have:

(a) $P^{[\overrightarrow{\text { odd }}, s]}(x)$ has a pole of order $m$ at $s=\lambda$ and $P^{[\overrightarrow{\operatorname{even}}, s]}(x)$ has a pole of order $m$ at $s=\lambda$. Any singular quasi- $\chi^{\lambda}$-invariant hyperfunction is given as a linear combination of

$$
\begin{array}{cc}
P_{-q}^{[\overrightarrow{\text { odd }}, \lambda]}(x) & (q=1,2, \ldots, m), \\
P_{-q}^{[\overrightarrow{\text { even }, \lambda]}(x)} & (q=1,2, \ldots, m) .
\end{array}
$$

(b) We have

$$
\begin{aligned}
\int P_{-q}^{[\overrightarrow{\text { odd }}, \lambda]}(x) \exp (-\sqrt{-1}\langle x, y\rangle) d x \\
=\sum_{\substack{l+r=-q, l \geq\lfloor n / 2\rfloor-2 m, r \geq m-\lfloor n / 2\rfloor}}(-1)^{r} A_{\text {odd }}(\lambda)_{l} P_{r}^{[\overrightarrow{\text { odd }},-\lambda-n]}(y)
\end{aligned}
$$

for $q=1,2, \ldots, m$ and

$$
\begin{aligned}
\int P_{-q}^{[\overrightarrow{\mathrm{even}}, \lambda]}(x) \exp (-\sqrt{-1}\langle x, y\rangle) d x \\
=\sum_{\substack{l+r=-q, l \geq\left\lfloor\frac{n+1}{2}\right\rfloor-2 m, r \geq m-\left\lfloor\frac{n+1}{2}\right\rfloor}}(-1)^{r} A_{\text {even }}(\lambda)_{l} P_{r}^{[\overrightarrow{\mathrm{even}},-\lambda-n]}(y)
\end{aligned}
$$

for $q=1,2, \ldots, m$.

2. Suppose that $\lambda$ is odd. We set $m:=-(\lambda-1) / 2$. Then we have

(a) $P^{[\overrightarrow{\text { odd }}, s]}(x)$ has a pole of order $m-1$ at $s=\lambda$ and $P^{[\overrightarrow{\mathrm{even}}, s]}(x)$ has a pole of order $m$ at $s=\lambda$. Any singular quasi- $\chi^{\lambda}$-invariant hyperfunction is given as a linear combination of

$$
\begin{array}{ll}
P_{-q}^{[\overrightarrow{\mathrm{dd}}, \lambda]}(x) & (q=1,2, \ldots, m-1), \\
P_{-q}^{[\mathrm{even}, \lambda]}(x) & (q=1,2, \ldots, m) .
\end{array}
$$


(b) We have

$$
\begin{gathered}
\int P_{-q}^{[\overrightarrow{\mathrm{odd}}, \lambda]}(x) \exp (-\sqrt{-1}\langle x, y\rangle) d x \\
=\sum_{\substack{l+r=-q, l \geq\left\lfloor\frac{n+1}{2}\right\rfloor-2 m+1, r \geq m-\left\lfloor\frac{n+1}{2}\right\rfloor}}(-1)^{r} A_{o d d}(\lambda)_{l} P_{r}^{[\overrightarrow{\mathrm{odd}},-\lambda-n]}(y)
\end{gathered}
$$

for $q=1,2, \ldots, m-1$ and

$$
\begin{aligned}
\int P_{-q}^{[\overrightarrow{\mathrm{even}}, \lambda]}(x) \exp (-\sqrt{-1}\langle x, y\rangle) d x \\
=\sum_{\substack{l+r=-q, l \geq\left\lfloor\frac{n+2}{2}\right\rfloor-2 m \\
r \geq m-\left\lfloor\frac{n+2}{2}\right\rfloor}}(-1)^{r} A_{\text {even }}(\lambda)_{l} P_{r}^{[\overrightarrow{\operatorname{even}},-\lambda-n]}(y)
\end{aligned}
$$

for $q=1,2, \ldots, m$.

Proof. The statements in 1-(a) and 2-(a) are proved as direct consequences of Theorem 6.3. Namely, suppose that $\lambda$ is in $-n+1 \leq \lambda \leq-1$. By (54), if $\lambda$ is an even integer, then $P^{[\overrightarrow{\text { odd }}, s]}(x)$ and $P^{[\overrightarrow{\text { even }, s]}}(x)$ have poles of order $m=-\lambda / 2$ at $s=\lambda$. By (56), if $\lambda$ is an odd integer, then $P^{[\overrightarrow{\text { odd }}, s]}(x)$ and $P^{[\overrightarrow{\text { even }}, s]}(x)$ have poles of order $m-1=-(\lambda-1) / 2-1$ and $m=-(\lambda-1) / 2$ at $s=\lambda$, respectively. Therefore, by Proposition 4.2 , we have 1 -(a) and $2-(\mathrm{a})$.

Next we go to the proof of 1-(b) and 2-(b). Remember the formula of the Fourier transform of $P^{[\overrightarrow{\text { odd }}, s]}(x)$ (resp. $P^{[\overrightarrow{\text { even }}, s]}(x)$ ) given by

$$
\begin{gathered}
\int P^{[\overrightarrow{\mathrm{odd}}, s]}(x) \exp (-\sqrt{-1}\langle x, y\rangle) d x=A_{\text {odd }}(s) P^{[\overrightarrow{\mathrm{odd}},-s-n]}(y) \\
\left(\text { resp. } \int P^{[\overrightarrow{\mathrm{even}}, s]}(x) \exp (-\sqrt{-1}\langle x, y\rangle) d x=A_{\text {even }}(s) P^{[\overrightarrow{\mathrm{even}},-s-n]}(y)\right)
\end{gathered}
$$

in Proposition 9.1. We expand the both hand sides of (117) into the Laurent 
series with respect to $s \in \mathbb{C}$ at $s=\lambda$.

(118)

$$
\begin{gathered}
\sum_{q \in \mathbb{Z}}(s-\lambda)^{-q} \int P_{-q}^{[\overrightarrow{\text { odd }}, \lambda]}(x) \exp (-\sqrt{-1}\langle x, y\rangle) d x \\
=\sum_{q \in \mathbb{Z}}(s-\lambda)^{-q} \sum_{\substack{l+r=-q \\
l, r \in \mathbb{Z}}}(-1)^{r} A_{\text {odd }}(\lambda)_{l} P_{r}^{[\overrightarrow{\text { odd }},-\lambda-n]}(y) \\
\text { resp. } \sum_{q \in \mathbb{Z}}(s-\lambda)^{-q} \int P_{-q}^{[\overrightarrow{\operatorname{even}}, \lambda]}(x) \exp (-\sqrt{-1}\langle x, y\rangle) d x \\
\left.=\sum_{q \in \mathbb{Z}}(s-\lambda)^{-q} \sum_{\substack{l+r=-q \\
l, r \in \mathbb{Z}}}(-1)^{r} A_{\text {even }}(\lambda)_{l} P_{r}^{[\overrightarrow{\text { even }},-\lambda-n]}(y)\right)
\end{gathered}
$$

By comparing the Laurent expansion coefficients on the both hand sides of (118), we have

$$
\begin{gathered}
\int P_{-q}^{[\overrightarrow{\mathrm{odd}}, \lambda]}(x) \exp (-\sqrt{-1}\langle x, y\rangle) d x \\
=\sum_{\substack{l+r=-q \\
l, r \in \mathbb{Z}}}(-1)^{r} A_{\mathrm{odd}}(\lambda)_{l} P_{r}^{[\overrightarrow{\mathrm{odd}},-\lambda-n]}(y) \\
\left(\text { resp. } \int P_{-q}^{[\overrightarrow{\mathrm{even}}, \lambda]}(x) \exp (-\sqrt{-1}\langle x, y\rangle) d x\right. \\
\left.=\sum_{\substack{l+r=-q \\
l, r \in \mathbb{Z}}}(-1)^{r} A_{\mathrm{even}}(\lambda)_{l} P_{r}^{[\overrightarrow{\operatorname{even}},-\lambda-n]}(y)\right)
\end{gathered}
$$

The sum $\sum_{\substack{l+r=-q \\ l, r \in \mathbb{Z}}}$ in the right hand side is a finite sum since $A_{\text {odd }}(\lambda)_{l}$ and $P_{r}^{[\overrightarrow{\text { odd }},-\lambda-n]}(y)$ (resp. $A_{\text {even }}(\lambda)_{l}$ and $P_{r}^{[\overrightarrow{\operatorname{even}},-\lambda-n]}(y)$ ) are zero if $l$ and $r$ are sufficiently small. Indeed, the orders of poles of $A_{\text {odd }}(s)$ and $P^{[\overrightarrow{\text { odd }},-s-n]}(y)$ (resp. $A_{\text {even }}(s)$ and $\left.P^{[\overrightarrow{\text { even }},-s-n]}(y)\right)$ can be computed by the formula (107) and (108) and by the results (111) and (114), respectively. Namely, when $\lambda$ is an even integer, $A_{\text {odd }}(s)$ (resp. $\left.A_{\text {even }}(s)\right)$ has a pole of order

$$
\begin{gathered}
-l_{\text {odd }}=-\left\lfloor\frac{n}{2}\right\rfloor-\lambda=-\left\lfloor\frac{n}{2}\right\rfloor-2 m \\
\left(\text { resp. }-l_{\text {even }}=-\left\lfloor\frac{n+1}{2}\right\rfloor-\lambda=-\left\lfloor\frac{n+1}{2}\right\rfloor-2 m\right)
\end{gathered}
$$

at $s=\lambda$. Here, $l_{\text {odd }}$ and $l_{\text {even }}$ are the numbers defined by (109). On the other hand, we see that $P^{[\overrightarrow{\text { odd }},-s-n]}(y)$ (resp. $P^{[\overrightarrow{\mathrm{even}},-s-n]}(y)$ ) has a pole of 
order $m-\left\lfloor\frac{n}{2}\right\rfloor$ (resp. $\left.m-\left\lfloor\frac{n+1}{2}\right\rfloor\right)$ at $s=\lambda$ from the result 1 -(a) and 2-(a). Then, $A_{\text {odd }}(\lambda)_{l}$ and $P_{r}^{[\overrightarrow{\mathrm{odd}},-\lambda-n]}(y)$ (resp. $A_{\text {even }}(\lambda)_{l}$ and $\left.P_{r}^{[\overrightarrow{\mathrm{even}},-\lambda-n]}(y)\right)$ are zero if and only if

$$
\begin{aligned}
l & <\left\lfloor\frac{n}{2}\right\rfloor-2 m, \quad r<m-\left\lfloor\frac{n}{2}\right\rfloor \\
\text { (resp. } l & \left.<\left\lfloor\frac{n+1}{2}\right\rfloor-2 m, \quad r<m-\left\lfloor\frac{n+1}{2}\right\rfloor\right),
\end{aligned}
$$

and hence we have (112) (resp. (115)). Similarly, when $\lambda$ is an odd integer, $A_{\text {odd }}(\lambda)_{l}$ and $P_{r}^{[\overrightarrow{\text { odd }},-\lambda-n]}(y)$ (resp. $A_{\text {even }}(\lambda)_{l}$ and $P_{r}^{[\overrightarrow{\text { even }},-\lambda-n]}(y)$ ) are zero if and only if

$$
\begin{array}{rl}
l & l<\left\lfloor\frac{n+1}{2}\right\rfloor-2 m+1, \quad r<m-\left\lfloor\frac{n+1}{2}\right\rfloor \\
\text { (resp. } l & \left.l<\left\lfloor\frac{n+2}{2}\right\rfloor-2 m, \quad r<m-\left\lfloor\frac{n+2}{2}\right\rfloor\right),
\end{array}
$$

and hence we have (113) (resp. (116)).

THEOREM 9.4. (square matrices 2) Let $\boldsymbol{V}$ be the space of $n \times n$ real square matrices and let $\lambda$ be an integer in $\lambda \leq-n$. The functions $A_{\text {even }}(s)$ and $A_{\text {odd }}(s)$ are defined by (107) and (108). Then we have:

1. $P^{[\overrightarrow{\mathrm{odd}}, s]}(x)$ (resp. $\left.P^{[\overrightarrow{\mathrm{even}}, s]}(x)\right)$ has a pole of order o( $\left.\overrightarrow{\mathrm{odd}}, \lambda\right)$ (resp. $o(\overrightarrow{\text { even }}, \lambda))$ at $s=\lambda$. Any singular quasi- $\chi^{\lambda}$-invariant hyperfunction is given as a linear combination of

$$
\begin{array}{cc}
P_{-q}^{[\overrightarrow{\mathrm{odd}}, \lambda]}(x) & (q=1,2, \ldots, o(\overrightarrow{\mathrm{odd}}, \lambda)) \\
P_{-q}^{[\overrightarrow{\mathrm{even}}, \lambda]}(x) & (q=1,2, \ldots, o(\overrightarrow{\mathrm{even}}, \lambda))
\end{array}
$$

Here,

$$
\begin{aligned}
& o(\overrightarrow{\operatorname{odd}}, \lambda)= \begin{cases}n / 2 & \text { when } n \text { is even, } \\
(n+1) / 2 & \text { when } n \text { is odd and } \lambda \text { is even, } \\
(n-1) / 2 & \text { when } n \text { is odd and } \lambda \text { is odd. }\end{cases} \\
& o(\overrightarrow{\text { even }}, \lambda)= \begin{cases}n / 2 & \text { when } n \text { is even, } \\
(n+1) / 2 & \text { when } n \text { is odd and } \lambda \text { is odd, } \\
(n-1) / 2 & \text { when } n \text { is odd and } \lambda \text { is even. }\end{cases}
\end{aligned}
$$


2. We have

$$
\begin{aligned}
\int P_{-q}^{[\overrightarrow{\text { odd }}, \lambda]}(x) \exp (-\sqrt{-1}\langle x, y\rangle) d x \\
=\sum_{\substack{l+r=-q, l \geq l_{\text {odd }}, r \geq 0}}(-1)^{r} A_{\text {odd }}(\lambda)_{l} P_{r}^{[\overrightarrow{\text { odd }},-\lambda-n]}(y)
\end{aligned}
$$

for $q=1,2, \ldots, o(\overrightarrow{\mathrm{odd}}, \lambda)$ and

$$
\begin{aligned}
\int & P_{-q}^{[\overrightarrow{\mathrm{even}}, \lambda]}(x) \exp (-\sqrt{-1}\langle x, y\rangle) d x \\
= & \sum_{\substack{l+r=-q, l \geq l_{\text {even }}, r \geq 0}}(-1)^{r} A_{\text {even }}(\lambda)_{l} P_{r}^{[\overrightarrow{\mathrm{even}},-\lambda-n]}(y)
\end{aligned}
$$

for $q=1,2, \ldots, o(\overrightarrow{\mathrm{even}}, \lambda)$. Here $l_{\text {odd }}$ and $l_{\text {even }}$ have been defined by $(110)$.

Proof. Since we assume that $\lambda \leq-n$, we have (122) by Proposition 4.2 and by the orders of poles of $P^{[\overrightarrow{\text { odd }}, s]}(x)$ and $P^{[\overrightarrow{\mathrm{even}}, s]}(x)$ at $s=\lambda$. The order of poles have been computed in (58) and (60), and we have (123). Then we have the result of the first item from Proposition 4.2 .

Next we prove the formulas in the second item. The formulas of the Fourier transforms of the Laurent expansion coefficients $P_{-q}^{[\overrightarrow{\text { odd }}, \lambda]}(x)$ and $P_{-q}^{[\overrightarrow{\text { even }}, \lambda]}(x)$ are given by $(119)$. Since $P^{[\overrightarrow{\text { odd }},-s-n]}(y)$ and $P^{[\overrightarrow{\mathrm{even}},-s-n]}(y)$ are holomorphic at $s=\lambda$ if $\lambda \leq-n, P_{r}^{[\overrightarrow{\text { odd }},-\lambda-n]}(y)$ and $P_{r}^{[\overrightarrow{\text { even }},-\lambda-n]}(y)$ are zero if and only if $r<0$. The orders of poles of $A_{\text {odd }}(s)\left(\operatorname{resp} . A_{\text {even }}(s)\right)$ at $s=\lambda$ can be computed by the formulas in (107) (resp. (108)). Then we see that $A_{\text {odd }}(\lambda)_{l}$ (resp. $\left.A_{\text {even }}(\lambda)_{l}\right)$ is zero if $l<l_{\text {odd }}$ (resp. $l<l_{\text {even }}$ ). Here $l_{\text {odd }}$ (resp. $l_{\text {even }}$ ) is the number defined by (110). Then we have (124) and (125).

We call a singular tempered distribution $f(x)$ bi-singular if the Fourier transform $f^{\vee}(y)$ is also a singular tempered distribution. The author thinks that it is an interesting problem to ask if there exists a bi-singular $\boldsymbol{G}^{1}$ invariant tempered distribution on prehomogeneous vector spaces. The following corollary gives a necessary and sufficient condition for a Laurent expansion coefficients of $P^{[\vec{a}, s]}(x)$ to be bi-singular. 
Corollary 9.5. (square matrices 3) Let $\boldsymbol{V}$ be the space of $n \times n$ real square matrices. Let $i$ be a positive integer. Then, for $q=1,2, \ldots,\left\lfloor\frac{i+1}{2}\right\rfloor$, the Laurent expansion coefficient $P_{-q}^{[\overrightarrow{\text { even }},-i]}(x)$ is bi-singular if and only if

$$
\begin{cases}\left\lfloor\frac{n-1}{2}\right\rfloor \geq i-q \quad \text { if } i \text { is odd } \\ \left\lfloor\frac{n-2}{2}\right\rfloor \geq i-q \quad \text { if } i \text { is even. }\end{cases}
$$

We have

$$
\operatorname{Supp}\left(P_{-q}^{[\overrightarrow{\mathrm{even}},-i]}(x)\right)= \begin{cases}\overline{\boldsymbol{S}_{2 q-1}} & \text { if } i \text { is odd } \\ \overline{\boldsymbol{S}_{2 q}} & \text { if } i \text { is even }\end{cases}
$$

and

$$
\operatorname{Supp}\left(P_{-q}^{[\overrightarrow{\mathrm{even}},-i]}(x)^{\vee}\right)= \begin{cases}\overline{\boldsymbol{S}_{n-2 i+2 q-1}} & \text { if } i \text { is odd } \\ \overline{\boldsymbol{S}_{n-2 i+2 q}} & \text { if } i \text { is even } .\end{cases}
$$

For $q=1,2, \ldots,\left\lfloor\frac{i}{2}\right\rfloor$, the Laurent expansion coefficient $P_{-q}^{[\overrightarrow{\text { odd }},-i]}(x)$ is bisingular if and only if

$$
\begin{cases}\left\lfloor\frac{n-2}{2}\right\rfloor \geq i-q \quad \text { if } i \text { is odd } \\ \left\lfloor\frac{n-1}{2}\right\rfloor \geq i-q \quad \text { if } i \text { is even }\end{cases}
$$

We have

$$
\operatorname{Supp}\left(P_{-q}^{[\overrightarrow{\text { odd }},-i]}(x)\right)= \begin{cases}\overline{\boldsymbol{S}_{2 q-1}} & \text { if } i \text { is even } \\ \overline{\boldsymbol{S}_{2 q}} & \text { if } i \text { is odd }\end{cases}
$$

and

$$
\operatorname{Supp}\left(P_{-q}^{[\overrightarrow{\mathrm{even}},-i]}(x)^{\vee}\right)= \begin{cases}\overrightarrow{\boldsymbol{S}_{n-2 i+2 q-1}} & \text { if } i \text { is even } \\ \overline{\boldsymbol{S}_{n-2 i+2 q}} & \text { if } i \text { is odd }\end{cases}
$$

Proof. Suppose that $i$ is an even integer in $1 \leq i \leq n-1$. Then $P^{[\overrightarrow{\mathrm{even}}, s]}(x)$ has a pole of order $m=i / 2=\left\lfloor\frac{i+1}{2}\right\rfloor$ at $s=-i$ and the Fourier transform of the Laurent expansion coefficient $P_{-q}^{[\overrightarrow{\mathrm{ven}}, s]}(x)(q=$ $\left.1,2, \ldots,\left\lfloor\frac{i+1}{2}\right\rfloor\right)$ is given by (113). From this formula, the support of the Fourier transform of $P_{-q}^{[\overrightarrow{\mathrm{even}}, s]}(x)$ is contained in the singular set $\boldsymbol{S}$ if and only if the index $r$ in the right hand side of (113) is always negative. Since 
$l \geq\left\lfloor\frac{n+1}{2}\right\rfloor-2 m=\left\lfloor\frac{n+1}{2}\right\rfloor-i$, the minimum of $l$ is $\left\lfloor\frac{n+1}{2}\right\rfloor-i$. Then the maximum of $r=-l-q$ is $-\left\lfloor\frac{n+1}{2}\right\rfloor+i-q$. Then $-\left\lfloor\frac{n+1}{2}\right\rfloor+i-q \leq-1$ is a necessary and sufficient condition in order that the support of the Fourier transform of $P_{-q}^{[\overrightarrow{\mathrm{even}}, s]}(x)$ is contained in $\boldsymbol{S}$. This is equivalent to

$$
\left\lfloor\frac{n+1}{2}\right\rfloor-1=\left\lfloor\frac{n-1}{2}\right\rfloor \geq i-q .
$$

Next we suppose that $i$ is an odd integer in $1 \leq i \leq n-1$. Then $P^{[\overrightarrow{\mathrm{even}}, s]}(x)$ has a pole of order $m=(i+1) / 2=\left\lfloor\frac{i+1}{2}\right\rfloor$ at $s=-i$. The Fourier transform of the Laurent expansion coefficient $P_{-q}^{[\overrightarrow{\mathrm{even}}, s]}(x)(q=$ $\left.1,2, \ldots,\left\lfloor\frac{i+1}{2}\right\rfloor\right)$ is given by (116). From this formula, the support of the Fourier transform of $P_{-q}^{[\overrightarrow{\mathrm{even}}, s]}(x)$ is contained in the singular set $\boldsymbol{S}$ if and only if the index $r$ in the right hand side of (116) is always negative. Since $l \geq\left\lfloor\frac{n+2}{2}\right\rfloor-2 m=\left\lfloor\frac{n+2}{2}\right\rfloor-i-1$, the minimum of $l$ is $\left\lfloor\frac{n+2}{2}\right\rfloor-i-1$. Then the maximum of $r=-l-q$ is $-\left\lfloor\frac{n+2}{2}\right\rfloor+i+1-q$. Then $-\left\lfloor\frac{n+2}{2}\right\rfloor+i+1-q \leq-1$ is a necessary and sufficient condition in order that the support of the Fourier transform of $P_{-q}^{[\overrightarrow{\mathrm{even}}, s]}(x)$ is contained in $\boldsymbol{S}$. This is equivalent to

$$
\left\lfloor\frac{n+2}{2}\right\rfloor-2=\left\lfloor\frac{n-2}{2}\right\rfloor \geq i-q .
$$

By (132) and (133), we see that (126) is a necessary and sufficient condition in order that the Laurent expansion coefficient $P_{-q}^{[\overrightarrow{\mathrm{even}},-i]}(x)$ is bisingular for $q=1,2, \ldots,\left\lfloor\frac{i+1}{2}\right\rfloor$ and $i=1, \ldots, n-1$. In the similar way, we can prove that (129) is a necessary and sufficient condition in order that the Laurent expansion coefficient $P_{-q}^{[\overrightarrow{\text { odd }},-i]}(x)$ is bi-singular for $q=1,2, \ldots,\left\lfloor\frac{i}{2}\right\rfloor$ and $i=1, \ldots, n-1$.

Next we shall prove the support formulas. The formulas (127) and (130) are the consequences of (76), (78), (80) and (82).

We prove the formula (128). Suppose that $i$ is an even integer in $1 \leq$ $i \leq n-1$. Then we have

$$
\operatorname{Supp}\left(P_{-q}^{[\overrightarrow{\mathrm{even}},-i]}(x)^{\vee}\right)=\operatorname{Supp}\left(P_{-\left\lfloor\frac{n+1}{2}\right\rfloor+i-q}^{[\overrightarrow{\mathrm{even}}, i-n]}(y)\right)
$$

since $-\left\lfloor\frac{n+1}{2}\right\rfloor+i-q$ is the maximum number appearing in the index $r$ of the sum in the right hand side of (113). Indeed, $P_{-\left\lfloor\frac{n+1}{2}\right\rfloor+i-q}^{[\overrightarrow{\mathrm{even}, i-n]}}(y)$ has the 
largest support among the Laurent expansion coefficients in the right had side of (113). If $n$ is even, then $i-n$ is even, and we have

$$
\operatorname{Supp}\left(P_{-\left\lfloor\frac{n+1}{2}\right\rfloor+i-q}^{[\overrightarrow{\operatorname{even}},-n]}(y)\right)=\overline{\boldsymbol{S}_{-2\left(-\left\lfloor\frac{n+1}{2}\right\rfloor+i-q\right)}}=\overline{\boldsymbol{S}_{-2\left(-\frac{n}{2}+i-q\right)}}=\overline{\boldsymbol{S}_{n-2 i+2 q}},
$$

by (78). If $n$ is odd, then $i-n$ is odd, we have

$$
\begin{aligned}
\operatorname{Supp}\left(P_{-\left\lfloor\frac{n+1}{2}\right\rfloor+i-q}^{[\overrightarrow{\operatorname{even}}, i-n]}(y)\right) & =\overrightarrow{\boldsymbol{S}_{-2\left(-\left\lfloor\frac{n+1}{2}\right\rfloor+i-q\right)-1}}=\overrightarrow{\boldsymbol{S}_{-2\left(-\frac{n+1}{2}+i-q\right)-1}} \\
& =\overline{\boldsymbol{S}_{n-2 i+2 q}},
\end{aligned}
$$

by (76). Then we have

$$
\operatorname{Supp}\left(P_{-q}^{[\overrightarrow{\mathrm{even}},-i]}(x)^{\vee}\right)=\overline{\boldsymbol{S}_{n-2 i+2 q}},
$$

when $-i$ is an even integer. When $-i$ is an odd integer, we can prove in the same way that

$$
\operatorname{Supp}\left(P_{-q}^{[\overrightarrow{\mathrm{even}},-i]}(x)^{\vee}\right)=\operatorname{Supp}\left(P_{-\left\lfloor\frac{n+2}{2}\right\rfloor+i-q+1}^{[\overrightarrow{\mathrm{even}}, i-n]}(y)\right)=\overline{\boldsymbol{S}_{n-2 i+2 q-1}} .
$$

Thus we obtain the formula (128).

The formula (131) can be proved in the similar way as the proof of the formula (128).

The following corollary is the special case of Corollary 9.5.

Corollary 9.6. (square matrices 4) Let $\boldsymbol{V}$ be the space of $n \times n$ real square matrices and let $i$ be an integer in $1 \leq i \leq n-1$. Suppose that $f(x)$ is a $\chi^{-i}$-invariant tempered distribution on $\boldsymbol{V}$.

1. If $f(x)$ is even in the sense of Definition 2.2, then $f(x)$ is given as a constant multiple of $P_{-\left\lfloor\frac{i+1}{2}\right\rfloor}^{[\overrightarrow{\mathrm{even}},-i]}(x)$ and its Fourier transform $f^{\vee}(y)$ is a constant multiple of $P_{-\left\lfloor\frac{n-i+1}{2}\right\rfloor}^{[\overrightarrow{\mathrm{even}}, i-n]}(y)$. Then

$$
\operatorname{Supp}(f(x))=\overline{\boldsymbol{S}_{i}} \quad \text { and } \quad \boldsymbol{\operatorname { S u p p }}\left(f^{\vee}(y)\right)=\overline{\boldsymbol{S}_{n-i}} \text {. }
$$

2. If $f(x)$ is odd in the sense of Definition 2.2, then $f(x)$ is given as a constant multiple of $P_{-\left\lfloor\frac{i}{2}\right\rfloor}^{[\overrightarrow{\mathrm{odd}},-i]}(x)$ and its Fourier transform $f^{\vee}(y)$ is a constant multiple of $P_{\left.-\left\lfloor\frac{\mathrm{odd}}{2}\right\rfloor-n\right]}^{\overrightarrow{\mathrm{on}}\rfloor}(y)$. Then

$$
\operatorname{Supp}(f(x))=\overline{\boldsymbol{S}_{i-1}} \quad \text { and } \quad \boldsymbol{\operatorname { S u p p }}\left(f^{\vee}(y)\right)=\overline{\boldsymbol{S}_{n-i-1}} \text {. }
$$


Proof. We show only the first item. The second item can be proved in the same way.

We suppose that $-i$ is an even integer in $-n+1 \leq-i \leq-1$. We have stated in Proposition 4.1 that any $\chi^{-i}$-invariant hyperfunction can be written as a finite sum of Laurent expansion coefficients $P_{-o(\vec{a},-i)}^{[\vec{a},-i]}(x)$ $\left(\vec{a} \in \mathbb{C}^{2}\right)$. Every vector $\vec{a} \in \mathbb{C}^{2}$ can be written as

$$
\vec{a}=a \cdot \overrightarrow{\text { even }}+b \cdot \overrightarrow{\text { odd }}
$$

with some $a, b \in \mathbb{C}$. If $\vec{a} \neq 0$. Then we have

$$
o(\vec{a},-i)=o(\overrightarrow{\text { even }},-i)=o(\overrightarrow{\text { odd }},-i)=\frac{i}{2}
$$

by (54) in Theorem 6.3. Then we have

$$
P_{-o(\vec{a},-i)}^{[\vec{a},-i]}(x)=a \cdot P_{-\frac{i}{2}}^{[\overrightarrow{\mathrm{even}},-i]}(x)+b \cdot P_{-\frac{i}{2}}^{[\overrightarrow{\mathrm{odd}},-i]}(x),
$$

and hence every $\chi^{-i}$-invariant hyperfunction can be written as a linear combination of $P_{-\frac{i}{2}}^{[\mathrm{even},-i]}(x)$ and $P_{-\frac{i}{2}}^{[\overrightarrow{\mathrm{odd}},-i]}(x)$. In particular, if it is an even function, then it can be written as a constant multiplication of $P_{-\frac{i}{2}}^{[\overrightarrow{\mathrm{even}},-i]}(x)=$ $P_{-\left[\frac{i+1}{2}\right\rfloor}^{[\overrightarrow{\mathrm{even}},-i]}(x)$. According to the formula of the Fourier transform (113), the Fourier transform of $P_{-\frac{i}{2}}^{[\overrightarrow{\mathrm{even}},-i]}(x)=P_{-\left\lfloor\frac{i+1}{2}\right\rfloor}^{[\overrightarrow{\mathrm{even}},-i]}(x)$ is given as a constant multiplication of $P_{-\frac{i}{2}-\left\lfloor\frac{n+1}{2}\right\rfloor}^{[\overrightarrow{\mathrm{even}}, i-n]}(y)=P_{-\left\lfloor\frac{n-i+1}{2}\right\rfloor}^{[\overrightarrow{\mathrm{even}}, i-n]}(y)$.

Next we suppose that $-i$ is an odd integer in $-n+1 \leq-i \leq-1$. Then, by (56) in Theorem 6.3 , we have

$$
o(\vec{a},-i)= \begin{cases}\frac{i+1}{2} & \text { if } \vec{a} \notin A_{\mathrm{odd}}, \\ \frac{i-1}{2} & \text { if } \vec{a} \in A_{\mathrm{odd}} .\end{cases}
$$

For a vector $\vec{a}=a \cdot \overrightarrow{\text { even }}+b \cdot \overrightarrow{\text { odd }}$, we have

$$
P_{-o(\vec{a},-i)}^{[\vec{a},-i]}(x)=a \cdot P_{-\frac{i+1}{2}}^{[\overrightarrow{\mathrm{even}},-i]}(x)
$$

if $a \neq 0$, and

$$
P_{-o(\vec{a},-i)}^{[\vec{a},-i]}(x)=b \cdot P_{-\frac{i-1}{2}}^{[\overrightarrow{\mathrm{odd}},-i]}(x),
$$


if $a=0$. Then every $\chi^{-i}$-invariant hyperfunction can be written as a linear combination of $P_{-\frac{i+1}{2}}^{[\overrightarrow{\mathrm{even}},-i]}(x)$ and $P_{-\frac{i-1}{2}}^{[\overrightarrow{\mathrm{odd}},-i]}(x)$. In particular, if it is an even function, then it can be written as a constant multiplication of $P_{-\frac{i+1}{2}}^{[\overrightarrow{\mathrm{even}},-i]}(x)=P_{-\left\lfloor\frac{i+1}{2}\right\rfloor}^{[\overrightarrow{\mathrm{even}},-i]}(x)$. According to the formula of the Fourier transform $(116)$, the Fourier transform of $P_{-\frac{i+1}{2}}^{[\overrightarrow{\mathrm{even}},-i]}(x)=P_{-\left\lfloor\frac{i+1}{2}\right\rfloor}^{[\overrightarrow{\mathrm{even}},-i]}(x)$ is given as a constant multiplication of $P_{-\frac{i+1}{2}-\left\lfloor\frac{n+2}{2}\right\rfloor}^{[\overrightarrow{\mathrm{even}}, i-n]}(y)=P_{-\left\lfloor\frac{n-i+1}{2}\right\rfloor}^{[\overrightarrow{\mathrm{even}, i-n]}}(y)$.

After all, we see that, for any integer $-i$ in $-n+1 \leq-i \leq-1$, every even $\chi^{-i}$-invariant hyperfunction $f(x)$ can be written as a constant multiplication of $P_{-\left\lfloor\frac{i+1}{2}\right\rfloor}^{[\overrightarrow{\operatorname{even}},-i]}(x)$ and its Fourier transform $f^{\vee}(y)$ is given by a constant multiplication of $P_{-\left\lfloor\frac{n-i+1}{2}\right\rfloor}^{[\overrightarrow{\mathrm{even}, i-n]}}(y)$. We see that $\operatorname{Supp}(f(x))=\overline{\boldsymbol{S}_{i}}$ and $\operatorname{Supp}\left(f^{\vee}(y)\right)=\overline{\boldsymbol{S}_{n-i}}$ by Theorem 7.1. Thus we complete the proof of the first item.

The second item can be proved by utilizing (54), (56) in Theorem 6.3 and (112), (115).

Remark 9.1. We can prove the following fact: if $f(x)$ is $\chi^{-i}$-invariant and $\operatorname{Supp}(f(x))=\overline{\boldsymbol{S}_{i}}$, then $f(x)$ is a tempered distribution given as a $\boldsymbol{G}^{1}$-invariant measure on $\boldsymbol{S}_{i}$, and hence $f(x)$ is an even tempered distribution. On the other hand, by taking $f(x)=P_{-\left\lfloor\frac{i}{2}\right\rfloor}^{[\overrightarrow{\text { odd }},-i]}(x)$, we have $f(x)$ is $\chi^{-i}$-invariant and $\operatorname{Supp}(f(x))=\overline{\boldsymbol{S}_{i-1}}$. However, even if $f(x)$ is $\chi^{-i}$ invariant and $\operatorname{Supp}(f(x))=\overline{\boldsymbol{S}_{i-1}}$, it is not derived that $f(x)$ is an odd tempered distribution. Indeed, since there is an even $\chi^{-i}$-invariant tempered distribution $g(x)$ supported in $\boldsymbol{S}_{i}, f(x)+g(x)$ is $\chi^{-i}$-invariant and $\operatorname{Supp}(f(x)+g(x))=\overline{\boldsymbol{S}_{i-1}}$. But $f(x)+g(x)$ may not be an odd tempered distribution.

Next we consider the alternating matrix space. Let $\boldsymbol{V}$ be the space of $2 n \times 2 n$ alternating matrices. We define

$$
\begin{aligned}
\left\{\begin{array}{c}
A_{\text {even }}(s) \\
A_{\text {odd }}(s)
\end{array}\right\}:= & (2 \pi)^{n(n-1)} \times(-1)^{n(n+1) / 2} \\
& \times \Gamma(s+1) \Gamma(s+3) \cdots \Gamma(s+2 n-1) \times(\sqrt{-1})^{n} \\
& \times\left\{\begin{array}{l}
(\exp (-\pi \sqrt{-1} s / 2)-\exp (\pi \sqrt{-1} s / 2))^{n} \\
(\exp (-\pi \sqrt{-1} s / 2)+\exp (\pi \sqrt{-1} s / 2))^{n}
\end{array}\right\} .
\end{aligned}
$$


We denote by $A_{\text {odd }}(\lambda)_{l}$ (resp. $\left.A_{\text {even }}(\lambda)_{l}\right)$ the coefficients of the Laurent series expansions $A_{\text {odd }}(s)=\sum_{l \in \mathbb{Z}} A_{\text {odd }}(\lambda)_{l}(s-\lambda)^{l}\left(\right.$ resp. $A_{\text {even }}(s)=$ $\left.\sum_{l \in \mathbb{Z}} A_{\text {even }}(\lambda)_{l}(s-\lambda)^{l}\right)$.

We suppose that $\lambda$ is a negative integer. When $-2 n+2 \leq \lambda \leq-1$, it is easily seen that $A_{\text {odd }}(s)$ (resp. $A_{\text {even }}(s)$ ) has a pole of order $-l_{\text {odd }}$ (resp. $\left.-l_{\text {even }}\right)$ at $s=\lambda$ where

$$
\begin{gathered}
l_{\text {odd }}:= \begin{cases}\left\lfloor\frac{\lambda}{2}\right\rfloor & \text { if } \lambda \text { is an even integer, } \\
n+\left\lfloor\frac{\lambda}{2}\right\rfloor & \text { if } \lambda \text { is an odd integer. }\end{cases} \\
\text { (resp. } l_{\text {even }}:=\left\{\begin{array}{ll}
n+\left\lfloor\frac{\lambda}{2}\right\rfloor & \text { if } \lambda \text { is an even integer, } \\
\left\lfloor\frac{\lambda}{2}\right\rfloor & \text { if } \lambda \text { is an odd integer. }
\end{array}\right)
\end{gathered}
$$

When $\lambda \leq-2 n+1, A_{\text {odd }}(s)$ (resp. $A_{\text {even }}(s)$ ) has a pole of order $-l_{\text {odd }}$ (resp. $\left.-l_{\text {even }}\right)$ at $s=\lambda$ where

$$
\begin{gathered}
l_{\text {odd }}:= \begin{cases}n & \text { if } \lambda \text { is an even integer } \\
0 & \text { if } \lambda \text { is an odd integer. }\end{cases} \\
\text { (resp. } l_{\text {even }}:=\left\{\begin{array}{ll}
0 & \text { if } \lambda \text { is an even integer } \\
n & \text { if } \lambda \text { is an odd integer. }
\end{array}\right)
\end{gathered}
$$

THEOREM 9.7. (alternating matrices 1) Let $\boldsymbol{V}$ be the space of $2 n \times 2 n$ alternating matrices and let $\lambda$ be an integer in $-2 n+2 \leq \lambda \leq-1$. The functions $A_{\text {even }}(s)$ and $A_{\text {odd }}(s)$ are defined by (134). When $\lambda$ is an even integer (resp. odd integer), we set $m:=-\lambda / 2($ resp. $m:=-(\lambda-1) / 2)$. Then we have:

1. $P^{[\overrightarrow{\mathrm{odd}}, s]}(x)$ (resp. $\left.P^{[\overrightarrow{\mathrm{even}}, s]}(x)\right)$ has a pole of order $m$ at $s=\lambda$ and $P^{[\overrightarrow{\text { even, }}, s]}(x)$ (resp. $\left.P^{[\overrightarrow{\text { odd }}, s]}(x)\right)$ is non-zero and holomorphic at $s=\lambda$. Any singular quasi- $\chi^{\lambda}$-invariant hyperfunction is given as a linear combination of

$$
P_{-q}^{[\overrightarrow{\mathrm{odd}}, \lambda]}(x) \quad\left(\text { resp. } P_{-q}^{[\overrightarrow{\mathrm{even}}, \lambda]}(x)\right)
$$

with $q=1,2, \ldots, m$. 
2. We have

$$
\begin{gathered}
\int P_{-q}^{[\overrightarrow{\text { odd }}, \lambda]}(x) \exp (-\sqrt{-1}\langle x, y\rangle) d x \\
=\sum_{r=0}^{m-q}(-1)^{r} A_{\text {odd }}(\lambda)_{-q-r} P_{r}^{[\overrightarrow{\text { odd }},-\lambda-2 n+1]}(y) \\
\left(\text { resp. } \int P_{-q}^{[\overrightarrow{\mathrm{even}}, \lambda]}(x) \exp (-\sqrt{-1}\langle x, y\rangle) d x\right. \\
\left.=\sum_{r=0}^{m-q}(-1)^{r} A_{\text {even }}(\lambda)_{-q-r} P_{r}^{[\overrightarrow{\operatorname{even}},-\lambda-2 n+1]}(y)\right)
\end{gathered}
$$

for $q=1,2, \ldots, m$.

Proof. The orders of poles of $P^{[\overrightarrow{\text { odd }}, s]}(x)$ and $P^{[\overrightarrow{\mathrm{even}}, s]}(x)$ are derived from (67). Then we have the first item from Proposition 4.2.

We go to the proof of the second item. Let $\lambda$ be an even (resp. odd) integer in $-2 n+2 \leq \lambda \leq-1$ and let $m:=-\lambda / 2$ (resp. $m:=-(\lambda-1) / 2)$. Consider the formulas of the Fourier transform of the Laurent expansion coefficients $P_{-q}^{[\overrightarrow{\text { odd }}, \lambda]}(x)$ (resp. $P_{-q}^{[\overrightarrow{\text { even }}, \lambda]}(x)$ ),

$$
\begin{gathered}
\int P_{-q}^{[\overrightarrow{\mathrm{odd}}, \lambda]}(x) \exp (-\sqrt{-1}\langle x, y\rangle) d x \\
=\sum_{\substack{l+r=-q \\
l, r \in \mathbb{Z}}}(-1)^{r} A_{\text {odd }}(\lambda)_{l} P_{r}^{[\overrightarrow{\text { odd }},-\lambda-n]}(y) . \\
\left(\text { resp. } \int P_{-q}^{[\overrightarrow{\operatorname{even}}, \lambda]}(x) \exp (-\sqrt{-1}\langle x, y\rangle) d x\right. \\
\left.=\sum_{\substack{l+r=-q \\
l, r \in \mathbb{Z}}}(-1)^{r} A_{\text {even }}(\lambda)_{l} P_{r}^{[\overrightarrow{\text { even }},-\lambda-n]}(y) .\right)
\end{gathered}
$$

Then $A_{\text {odd }}(s)$ (resp. $\left.A_{\text {even }}(s)\right)$ has a pole of order $-l_{\text {odd }}\left(\right.$ resp. $\left.-l_{\text {even }}\right)$ at $s=\lambda$. Here, $l_{\text {odd }}$ and $l_{\text {even }}$ are defined by (135). On the other hand, we see that $P^{[\overrightarrow{\text { odd }},-s-n]}(y)$ (resp. $P^{[\overrightarrow{\text { even }},-s-n]}(y)$ ) is holomorphic at $s=\lambda$ as we have proved in the first item. Then we have $A_{\text {odd }}(\lambda)_{l}$ and $P_{r}^{[\overrightarrow{\text { odd }},-\lambda-n]}(y)$ (resp. $A_{\text {even }}(\lambda)_{l}$ and $\left.P_{r}^{[\overrightarrow{\operatorname{even}},-\lambda-n]}(y)\right)$ are zero if

$$
\left.l<-l_{\text {odd }}=m \text { and } r<0 \quad \text { (resp. } l<-l_{\text {even }}=m \text { and } r<0\right),
$$

and hence we have (138). 
THEOREM 9.8. (alternating matrices 2) Let $\boldsymbol{V}$ be the space of $2 n \times 2 n$ alternating matrices and let $\lambda$ be an integer in $\lambda \leq-2 n+1$. The functions $A_{\text {even }}(s)$ and $A_{\text {odd }}(s)$ are defined by (134). Then we have:

1. $P^{[\overrightarrow{\mathrm{odd}}, s]}(x)\left(\right.$ resp. $\left.P^{[\overrightarrow{\mathrm{even}}, s]}(x)\right)$ has a pole of order $n$ at $s=\lambda$ and $P^{[\overrightarrow{\mathrm{even}}, s]}(x)\left(\right.$ resp. $\left.P^{[\overrightarrow{\mathrm{odd}}, s]}(x)\right)$ is non-zero and holomorphic at $s=\lambda$. Any singular quasi- $\chi^{\lambda}$-invariant hyperfunction is given as a linear combination of

$$
P_{-q}^{[\overrightarrow{\text { odd }}, \lambda]}(x) \quad\left(\text { resp. } P_{-q}^{[\overrightarrow{\mathrm{even}}, \lambda]}(x)\right)
$$

with $q=1,2, \ldots, n$.

2. We have

$$
\begin{gathered}
\int P_{-q}^{[\overrightarrow{\mathrm{odd}}, \lambda]}(x) \exp (-\sqrt{-1}\langle x, y\rangle) d x \\
=\sum_{r=0}^{n-q}(-1)^{r} A_{\text {odd }}(\lambda)_{-q-r} P_{r}^{[\overrightarrow{\mathrm{odd}},-\lambda-2 n+1]}(y) \\
\text { (resp. } \int P_{-q}^{[\overrightarrow{\mathrm{even}}, \lambda]}(x) \exp (-\sqrt{-1}\langle x, y\rangle) d x \\
\left.=\sum_{r=0}^{n-q}(-1)^{r} A_{\text {even }}(\lambda)_{-q-r} P_{r}^{[\overrightarrow{\mathrm{even}},-\lambda-2 n+1]}(y)\right)
\end{gathered}
$$

for $q=1,2, \ldots, n$.

Proof. The orders of poles of $P^{[\overrightarrow{\mathrm{odd}}, s]}(x)$ and $P^{[\overrightarrow{\mathrm{even}}, s]}(x)$ are derived from (69). Then we have the first item from Proposition 4.2. The second item can be proved in the same way as the proof of that of Theorem 9.7 by using $l_{\text {odd }}$ and $l_{\text {even }}$ given in (136).

The following corollary is a direct consequence of Theorems 9.7 and 9.8.

Corollary 9.9. (alternating matrices 3) Let $\boldsymbol{V}$ be the space of $2 n \times$ $2 n$ real alternating matrices. Then there are no bi-singular $\boldsymbol{G}^{1}$-invariant tempered distributions on $\boldsymbol{V}$. 


\section{REFERENCES}

[1] I. N. Bernstein, The analytic continuation of generalized functions with respect to a parameter, Functional Anal. Appl., 6 (1972), 26-40.

[2] I. M. Gelfand and G. E. Shilov, Generalized functions - properties and operations, Generalized Functions, vol. 1, Academic Press, New York and London, 1964.

[3] A. Gyoja, Bernstein-Sato's polynomial for several analytic functions, J. Math. Kyoto Univ., 33 (1993), no. 2, 399-411.

[4] Local b-functions of prehomogeneous Lagrangians, J. Math. Kyoto Univ., 33 (1993), no. 2, 413-436.

[5] J. Igusa, An introduction to the theory of local zeta functions, Studies in Advanced Mathematics, vol. 14, American Mathematical Society, 2000.

[6] M. Kashiwara, T. Kawai, and T. Kimura, Daisuukaisekigaku no Kiso (Foundations of Algebraic Analysis), Kinokuniya, Tokyo, 1980 (Japanese); The English translation was published by Princeton UP in 1985.

[7] M. Kashiwara and T. Miwa, Microlocal calculus and Fourier transforms of relative invariants of prehomogeneous vector spaces, Sûrikaisekikenkyûsho Kôkyûroku, $\mathbf{2 8 3}$ (1974), 60-147.

[8] M. Muro, Microlocal analysis and calculations on some relatively invariant hyperfunctions related to zeta functions associated with the vector spaces of quadratic forms, Publ. Res. Inst. Math. Sci. Kyoto Univ. (1986), no. 3, 395-463.

[9] - Singular invariant tempered distributions on regular prehomogeneous vector spaces, J. Funct. Anal., 76 (1988), no. 2, 317-345.

[10] - Invariant hyperfunctions on regular prehomogeneous vector spaces of commutative parabolic type, Tôhoku Math. J. (2), 42 (1990), no. 2, 163-193.

[11] Singular invariant hyperfunctions on the space of real symmetric matrices, Tôhoku Math. J. (2), 51 (1999), 329-364.

[12] - Singular invariant hyperfunctions on the space of complex and quaternion hermitian matrices, J. Math. Soc. Japan, 53 (2001), no. 3, 589-602.

[13] - Invariant hyperfunction solutions to invariant differential equations on the space of real symmetric matrices, J. Funct. Anal., 193 (2002), no. 2, 346-384.

[14] M. Raïs, Distributions homogènes sur des espaces de matrices, 30 (1972), Bull. Soc. Math. France, 5-109.

[15] — Identités de Capelli et distributions invariantes (I), preprint, Université de Poitiers (1986).

[16] S. Rallis and G. Schiffmann, Distributions invariantes par le groupe orthogonal, Lecture Note in Math. (Springer), 497 (1975), pp. 494-642.

[17] H. Rubenthaler, Distributions bi-invariantes par $S L_{n}(k)$, Lecture Note in Math. (Springer), 497 (1975), pp. 383-493.

[18] H. Saito, Explicit form of the zeta functions of prehomogeneous vector spaces, Math. Ann., 315 (1998), 587-615.

[19] I. Satake, On zeta functions associated with self-dual homogeneous cone, Number theory and Related Topics (Bombay) (S. Raghavan, ed.), Tata Institute of Fundamental Research, Tata Institute of Fundamental Research and Oxford UP (1989), 
pp. $177-193$.

[20] M. Sato and T. Shintani, On zeta functions associated with prehomogeneous vector spaces, Ann. of Math. (2), 100 (1974), 131-170.

[21] T. Shintani, On zeta functions associated with the vector spaces of quadratic forms, J. Fac. Sci. Univ. Tokyo Sect. IA Math., 22 (1975), 25-65.

[22] H. Weyl, The classical groups, Princeton University Press, 1946.

Gifu University

Yanagito 1-1

Gifu, 501-1193

Japan

muro@cc.gifu-u.ac.jp 